\title{
POSTPRINT
}

\author{
Katharina König \\ Florence Oloff
}

\section{Die Multimodalität alltagspraktischen Erzählens}

Zusammenfassung: Dieser Beitrag widmet sich der Analyse des Zusammenspiels sprachlich-hörbarer und sichtbar-kinesischer Praktiken, die beim alltäglichen Erzählen eingesetzt werden. Im Rahmen einer konversationsanalytisch basierten Untersuchung von Videoaufnahmen deutscher Alltagsgespräche wird die Bandbreite alltäglicher narrativer Praktiken in der face-to-face-Kommunikation aufgezeigt. Dies erfolgt exemplarisch anhand zweier Beispiele, in denen Einstieg, Ausgestaltung sowie Beendigung der Erzählung unter unterschiedlichen sequentiellen und multimodalen Bedingungen vollzogen werden. Die Untersuchung unterstreicht einerseits die Indexikalität alltäglicher narrativer Praktiken, andererseits die Notwendigkeit einer interaktionalen Narratologie, die diese Praktiken als Produkt sprachlicher, verkörperter und räumlicher Ressourcen sowie der Zusammenarbeit mehrerer Teilnehmer analysiert und konzeptualisiert.

Schlüsselwörter: Konversationelles Erzählen, Soziale Interaktion, Konversationsanalyse, Multimodalität, Videodaten, Alltagsgespräche, Kollaboratives Erzählen, Interaktionale Narratologie

\section{The Multimodality of Everyday Storytellings}

Abstract: This contribution investigates the interplay between verbal/audible and visible/embodied practices that are used in everyday storytelling. Using a conversation analytic approach to video recorded ordinary conversations in German, we aim

\footnotetext{
K. König

Germanistisches Institut, WWU Münster, Münster, Deutschland

E-Mail: katharina.koenig@wwu.de

F. Oloff

Faculty of Humanities, University of Oulu, P.O. Box 1000, Oulu, Finnland

E-Mail: florence.oloff@oulu.fi
} 
to illustrate the wide range of everyday narrative practices in face-to-face encounters. On the basis of two exemplary cases, the analysis considers how storytelling is started, carried out and brought to an end under different sequential and multimodal conditions. On the one hand, our analysis emphasizes the indexicality of mundane narrative practices, on the other hand, it shows the necessity of an interactional narratology framework that conceptualises these practices as resulting from the use of linguistic, embodied and spatial resources, as well as from the joint and coordinated interaction of multiple participants.

Keywords: conversational storytelling, social interaction, conversation analysis, multimodality, video data, mundane conversation, collaborative storytelling, interactional narratology

\section{Erzählen vom Alltag, Erzählen im Alltag}

Mündliches Erzählen ist eine fest in unseren kommunikativen Alltag integrierte Praktik: Durch Erzählen lassen wir andere an zeitlich zurückliegenden Situationen teilhaben; geteilte Erinnerungen werden festgehalten oder reaktiviert. Dabei ist Erzählen stets auf die Zuhörenden ausgerichtet und in seiner Ausgestaltung auf das Wissen und die (angenommenen) Werthaltungen der AdressatInnen zugeschnitten. Erzählen ist somit eine soziale Alltagspraktik par excellence.

Im Sinne Gunkels (1963 [1925]) hat Erzählen einen >Sitz im Leben<: Es handelt sich um eine Praktik, die nur in Bezug auf ihren jeweiligen Entstehens- und Gebrauchskontext erschlossen werden kann. Als rekonstruktive Gattung (vgl. Bergmann/Luckmann 1995) bearbeitet Erzählen zahlreiche rekurrente kommunikative Aufgaben (vgl. Sacks 1995, II 466ff.): Über die Praktik des Erzählens können sich die Interagierenden einer gemeinsamen Weltsicht versichern und Sozialität herstellen. Vorstellungen von Normen und moralischen Werten können ebenso zum Ausdruck gebracht werden (vgl. Bergmann 1987; Ochs/Taylor 1995) wie die Positionierung von Personen in der Erzählwelt und der erzählten Welt (vgl. Lucius-Hoene/ Deppermann 2004). Vermittels des Erzählens werden Identitäten zugeschrieben, eingenommen und hergestellt (vgl. de Fina 2006).

Die Konzepte des Erzählens und des Alltags stehen hierbei in verschiedenen Wechselverhältnissen (vgl. Gülich 2008): Zum einen werden im alltäglichen Erzählen Erlebnisse, Begebenheiten, Inhalte der als Alltag erfahrenen Lebenswelt zum Gegenstand der narrativen Rekonstruktion gemacht (»Erzählen vom Alltag «): SprecherInnen erzählen davon, was ihnen beim Einkaufen, auf dem Weg zur Arbeit oder im Restaurant passiert ist. Die narrativ dargestellten Erlebnisse sind somit im Kontext eines »usuelle[n] spontane[n] Tun[s] (vgl. Heinemann 2000, S. 604) verortet. Gleichzeitig besteht eine der zentralen kommunikativen Aufgaben beim konversationellen Erzählen darin, die Erzählwürdigkeit, die tellability (vgl. Ochs/Capps 2001; Sacks 1986) des Erlebten herauszustellen. Es wird also oftmals gerade etwas Überraschendes, etwas besonders Amüsantes oder etwas von der Erwartung Abweichendes zum Gegenstand einer narrativen Rekonstruktion gemacht. Welcher Aspekt des Erzählten jedoch als aus dem Alltäglichen herausgehoben dargestellt und als 
erzählwürdig behandelt wird, lässt sich nicht a priori in dem Erlebten selbst verorten, sondern wird erst im Kontext des Gesprächs zwischen den Interagierenden vorgenommen (vgl. Gülich 2008, S. 408).

Zum anderen ist die Praktik des Erzählens häufig in das Alltagsgeschehen eingebettet (»Erzählen im Alltag«): Man erzählt bei Treffen mit Freunden in der Bar, während man das Abendessen zubereitet oder während man mit seiner Familie im Auto sitzt. Eine solche das Alltagshandeln kennzeichnende Multiaktivität (vgl. Haddington et al. 2014) stellt einen grundlegenden Kontext dar, in den ein Erzählprozess integriert werden muss. Als Praktik im kommunikativen Alltag unterliegt Erzählen nicht den gleichen Funktionalisierungen wie etwa im institutionellen Diskurs (vgl. Gülich 1980); die Art und der Umfang seiner Ausgestaltung sowie die genaue zeitlich-sequentielle Positionierung eines Narrativs im Verlaufe einer Unterhaltung sind nicht festgelegt. Erzählen im Alltag entsteht aus der Situation heraus, es wird von den ZuhörerInnen lizensiert und mitgestaltet und findet zumeist in einem privat-vertrauten Rahmen statt, der wiederum spezifische Themen ermöglicht, die beim öffentlichinstitutionellen Erzählen nicht in gleichem Maße erwartbar wären (vgl. Mandelbaum 2013; Quasthoff 2001; Schütte 2001). Erzählen konstituiert somit selbst wiederum Alltag (vgl. Schütte 2001, S. 1487 zur Routinisierung bei Alltagsgesprächen).

Bisherige Studien zum mündlichen Alltagserzählen beziehen sich vornehmlich auf die Beschreibung sprachlicher und vokaler Verfahren (vgl. Gülich 2008, S. 409). Erzählen findet jedoch häufig unter den Bedingungen der räumlichen Ko-Präsenz der SprecherInnen statt, die den zusätzlichen Gebrauch von körperlichen und materiellen Ressourcen ermöglicht (vgl. C. Goodwin 1981; M. H. Goodwin 1991). Erzählen ist somit als körpergebundene und verkörperlichte Praktik zu verstehen, die in einem bestimmten Interaktionsraum situiert ist (vgl. Hausendorf 2010; Mondada 2013; Schmitt 2015). Wie Mimik, Gestik, Proxemik, aber auch räumliche Konstellationen und Objekte für die Zwecke des Erzählens genutzt werden, ist bislang nur in Ansätzen erforscht bzw. auf spezifische Phänomene oder sequentielle Positionen bezogen (vgl. Mandelbaum 2013, S. 501ff.). Es ist jedoch davon auszugehen, dass eine solche grundlegende Multimodalität kennzeichnend ist für die alltägliche Praxis des Erzählens in Ko-Präsenz. Der vorliegende Beitrag setzt sich daher zum Ziel, in einem gesprächsanalytischen Zugang die multiplen Aspekte der Kontextgebundenheit und Kontexthervorbringung von Erzählungen anhand der Analyse zweier exemplarischer Beispiele zu illustrieren. Dabei sollen Wege aufgezeigt werden, wie eine multimodale Erzählanalyse dazu beitragen kann, die tatsächliche Alltagspraxis des Erzählens umfassender zu erschließen.

\section{Zur Kontextgebundenheit des Erzählens}

In ihrem grundlegend alltagspraktischen Paradigma untersucht die ethnomethodologische Konversationsanalyse primär solche narrativen Einheiten, die in natürlichen, nicht elizitierten, spontanen Interaktionen hervorgebracht wurden (vgl. Mandelbaum 2013; Sidnell 2010, insbesondere Kapitel 9). Ihr primäres Interesse besteht in der Untersuchung der Prozesse der gemeinsamen Hervorbringung sozialer Ordnung mit alltäglichen, zumeist unhinterfragten Praktiken der Sinnerzeugung in »ordinary con- 
versation « (vgl. Jefferson 1988; Sacks 1986). Zentraler Gegenstand eines solchen Ansatzes ist die Frage, wie eine Erzählung als big package aus dem turn-by-turntalk zwischen den an der Interaktion beteiligten SprecherInnen realisiert werden kann (vgl. Quasthoff 2001). Erzählen wird also als sequentiell eingebettete Praktik verstanden, die in mehrfacher Hinsicht mit dem situationellen Kontext verwoben ist (vgl. Goodwin 2015; Mandelbaum 2013; Quasthoff 2001; vgl. auch die Beiträge von Hoffmann, Kotthoff sowie Quasthoff/Stude in diesem Band). Inwiefern Erzählen aus seinem jeweiligen Kontext hervorgeht bzw. selbst einen Kontext etabliert, soll im Folgenden mit Verweis auf zentrale interaktionale Forschungsarbeiten skizziert werden.

\subsection{Erzählen wird in einem bestimmten Gesprächskontext relevant gemacht}

Die Praktik des Erzählens bedarf einer koordinierten Vorbereitung: Ein Sprecher/ eine Sprecherin muss sich zunächst ein ticket für die Realisierung dieser größeren Diskurseinheit von den Zuhörenden einholen. Formal geschieht dies oftmals durch ein so genanntes story preface (vgl. Sacks 1971; vgl. auch Jefferson 1978). ${ }^{1}$ Erst wenn man sich der Aufmerksamkeit der relevanten AdressatInnen sicher ist und wenn die grundlegende Erzählwürdigkeit hergestellt ist (vgl. Ochs/Capps 2001), kann ein Redebeitrag narrativ ausgebaut werden. Erzählungen müssen nicht nur von der erzählenden Person eingeführt und relevant gemacht werden; immer wieder werden SprecherInnen im kommunikativen Alltag durch story prompts (vgl. Lerner 1992) wie »Erzähl doch mal.« oder »Und? Wie war's?« zum Erzählen aufgefordert (siehe auch Gülich 1980). Ebenso können Erzählungen als affiliative zweite Handlungszüge auf vorangegangene Erzählungen realisiert werden (vgl. Siromaa 2012; Selting 2012). Erzählungen können zudem auch durch den aktuellen Handlungskontext relevant gemacht werden: Als Belegerzählungen in Beschwerden (vgl. Günthner 1999) oder als Krankheitsnarrative in medizinischen Diagnosegesprächen (vgl. Halkowski 2007) kommt ihnen eine spezifische Funktion für die jeweils aktuell zu bearbeitende kommunikative Aufgabe zu (vgl. Kallmeyer 1981). Praktiken des Erzählens können also durch spezifische sequentielle Kontexte relevant gemacht werden, sie erlangen ihre spezifische Funktion im jeweiligen Gesprächskontext.

\subsection{Erzählen schafft den Kontext, in dem es interpretierbar wird}

Im Sinne des reflexiven Kontextbegriffs der interpretativen Soziolinguistik (vgl. Gumperz 1992) können Erzählungen zudem selbst die Kontexte erschaffen, in denen sie interpretiert und verstanden werden sollen. Dies ist etwa dann der Fall, wenn Erzählungen zur Herstellung einer spezifischen Interaktionsmodalität (vgl. Kallmeyer 1979) genutzt werden: So können Beschwerdeerzählungen etwa zur Konstruktion einer ernsten Modalität beitragen, in der die Erzählung wiederum erst als moralisie-

\footnotetext{
${ }^{1}$ Bei narrativen Kleinstformaten, sogenannten small stories (vgl. Bamberg 2007), muss eine solche ausgedehnte narrationseinleitende Sequenz nicht notwendigerweise realisiert werden. Das Wissen um in einer community of practice geteilte Kontextualisierungshinweise kann ausreichend sein, um eine Äußerung als Narrativ zu kennzeichnen (vgl. Georgakopoulou 2015).
} 
rende Handlung erkennbar und interpretierbar wird (vgl. Günthner 1999). Ebenso kann durch Erzählungen ein humoristischer Rahmen hergestellt werden, durch den die Wertung des rekonstruierten Erlebnisses als spaßhaft-ironisierend kontextualisiert wird (vgl. Kotthoff 1998). Wechselseitiges Erzählen kann zudem zur Erzeugung von »Alltag « beitragen. In diesem Sinne ist es nicht allein der gegebene Kontext des Alltags, der dazu beiträgt, dass erzählt wird, sondern durch die Praktik des Erzählens wird der Rahmen des Alltäglichen überhaupt erst erschaffen (vgl. Schütte 2001). So wird etwa durch ritualisiertes und phatisches Erzählen, mit dem man seine ZuhörerInnen an seinen eigenen Erlebnissen und Weltanschauungen teilhaben lässt, familiärer Alltag konstruiert (vgl. Keppler 1994) oder es werden Gruppenidentitäten etabliert und gefestigt (vgl. de Fina 2006; Georgakopoulou 2015).

\subsection{Erzählen wird in seiner Ausgestaltung auf die jeweilige Situation zugeschnitten und in Kollaboration mit den ZuhörerInnen hervorgebracht}

Die eingangs festgehaltene Charakterisierung des Erzählens als soziale Praxis hat weitreichende Konsequenzen für die Einbettung und Ausgestaltung einer Erzählung. Nicht nur müssen die ZuhörerInnen der erzählenden Person das Rederecht für einen multi-unit turn einräumen. Durch die Realisierung verschiedener Rückmeldesignale bestimmen ZuhörerInnen die sequentielle Struktur zudem maßgeblich mit (vgl. Duranti 1986; Stivers 2008). Auch in anderer Hinsicht sind sie an der Konstruktion narrativer Sequenzen beteiligt: Im Sinne eines recipient design (vgl. Sacks et al. 1974, S. 727) beziehen ErzählerInnen stets das (vermutete) Vorwissen der Zuhörenden mit ein. Erzählt man der gleichen Person zum zweiten Mal von einem Erlebnis, wird etwa der Grad der Detaillierung bestimmter Passagen einer Erzählung systematisch angepasst (vgl. Norrick 1997; Schumann et al. 2015). Verfügen die ZuhörerInnen über verschiedene Wissensbestände, ergeben sich hieraus spezifische Anforderungen für die erzählende Person (vgl. Goodwin 1986). ZuhörerInnen können zudem selbst zu Ko-Erzählenden (gemacht) werden (vgl. Lerner 1992; Mandelbaum 1987). Zeigen sie zum Ende einer Erzählung nicht die passende Reaktion, kann dies zu einer Expansion einer narrativen Sequenz führen (vgl. Selting 2012, 2017). Abschließend kann also festgehalten werden: »Es gibt alltagsweltlich in keinem Fall den >einsamen Erzähler«.«(Kallmeyer 1981, S. 420)

In einem interaktionalen Zugang wird alltägliches Erzählen als eine reflexive Praktik verstanden, die in einem spezifischen sequentiellen Kontext stattfindet und die selbst wiederum zur Situationsinterpretation beiträgt. Auch wenn die grundlegende Dialogizität sowie der sequentiell-prozessuale Aufbau von Erzählungen somit erfasst werden können, wird ein weiterer zentraler Kontextfaktor von Alltagspraktiken des Erzählens jedoch meist außer Acht gelassen: Zahlreiche narrative Rekonstruktionen werden in face-to-face-Gesprächen hervorgebracht. 


\section{Ansätze zu einer multimodalen Erzählanalyse}

In face-to-face-Gesprächen ist Erzählen als körpergebundene und verkörperlichte Praktik zu verstehen, die in einem bestimmten Interaktionsraum situiert ist (vgl. Hausendorf 2010; Mondada 2013; Schmitt 2015). Sowohl verschiedene Phasen des Erzählens als auch unterschiedliche Beteiligungsrollen können durch kinesische, materielle oder räumliche Praktiken abgegrenzt werden (vgl. Goodwin 1984; Müller 2003; Selting 2017). Ebenso können rekonstruierte Ereignisse redebegleitend oder auch ausschließlich körperlich re-enaktiert werden (vgl. Sidnell 2006; Thompson/ Suzuki 2014). Nicken und spezifischen Gesichtsausdrücken kommt als Hörerreaktion eine zentrale Bedeutung bei der Beendigung bzw. Expansion von Erzählungen zu (vgl. Ruusuvuori/Peräkylä 2009; Stivers 2008).

Diese Multimodalität alltagspraktischen Erzählens ist bislang jedoch nicht systematisch in eine interaktional-linguistische Narratologie integriert worden. Gülich und Mondada (2008, S. 115) sprechen von einem »Logozentrismus« der bisherigen konversationsanalytischen Forschung. Vorhandene Forschungsarbeiten zum Einsatz multimodaler Ressourcen in der face-to-face-Interaktion, die sich dezidiert mit Praktiken des Erzählens befassen, gehen meist nur auf spezifische Phasen des Erzählens (etwa der Erzähleinstieg bei Goodwin 1991; Markierungen der narrativen Klimax, vgl. Ford/Fox 2010; Selting 2017) oder ausgewählte Teilpraktiken (wie etwa die multimodale Ausgestaltung der Redewiedergabe, vgl. Ehmer 2011; Stec et al. 2016) ein. Eine umfassende Konzeptualisierung von Erzählungen als multimodaler Praktik, die den gesamten Verlauf narrativer Sequenzen betrachtet und verschiedene Teilgattungen des Erzählens miteinander kontrastiert, steht hingegen noch aus.

Auch wenn diese Lücke in dem vorliegenden Beitrag nicht zur Gänze geschlossen werden kann, sollen die folgenden Analysen ausgewählter narrativer Verfahren in zwei Alltagserzählungen die Komplexität der Koordination multimodaler Ressourcen bei der Einleitung, der Durchführung sowie dem Abschluss alltäglichen Erzählens illustrieren. ${ }^{2}$ Die ausgewählten Erzählungen sind beide humoristisch gerahmt, arbeiten ein vorher von den Gesprächsteilnehmern bereits behandeltes Thema aus und haben von den ErzählerInnen selbst erlebte Begebenheiten zum Gegenstand. Andererseits weisen beide Erzählungen deutliche Unterschiede in der multimodalen Ausgestaltung des Erzählten auf, so dass sie die Bandbreite der im Korpus gefundenen Verfahren exemplifizieren. Dies soll an kurzen, mit multimodalen Annotationen sowie Bildern angereicherten Auszügen schlaglichtartig aufgezeigt werden (für die Transkriptionskonventionen des Audiotranskripts s. Selting et al. 2009; für die

\footnotetext{
2 Das erste Beispiel (»Ausweis«) entstand 2014 im Rahmen eines Forschungsprojektes an der Universität Zürich (SNF Ambizione Nr. 148146) zu gemeinsamen Formulierungsaktivitäten in Alltagsgesprächen. Es handelt sich um ein 3,5-stündiges gemeinsames Essen unter vier befreundeten Arbeitskollegen (gefilmt mit zwei Kameras). Das zweite Beispiel (»Zitrone«) wurde 2016 als Teil eines Seminars an der WWU Münster für das Korpus Multimodale Interaktion (KoMI) erhoben. Die Aufnahme dokumentiert ca. 90 Minuten eines geselligen Beisammenseins dreier FreundInnen, wobei die Kamera in der Position eines vierten Teilnehmers installiert wurde. Für die Bereitstellung des Materials aus dem Korpus Multimodale Interaktion (KoMI) danken wir Pepe Droste. Die Eigennamen der TeilnehmerInnen (sowie im Gespräch erwähnte Eigennamen) wurden in allen Auszügen durch Pseudonyme ersetzt.
} 
multimodalen Annotationen s. Mondada 2014a ${ }^{3}, 2018$ bzw. die Transkriptionskonventionen am Ende dieses Beitrags). Um Variationen in der Nutzung körperlichvisueller Ressourcen kontrastieren zu können, orientieren sich die Analysen jeweils an den drei zentralen Erzählphasen, dem Einstieg, der Ausgestaltung sowie dem Ausstieg.

\subsection{Beispiel 1: »Ausweis«}

\subsubsection{Einstieg in die Erzählung}

Im Vorfeld des ersten Auszuges haben alle InteraktionsteilnehmerInnen die eigenen grauen Haare kommentiert. Marie kündigt daraufhin an, sich Tee einzuschenken, und bietet Tatiana ebenfalls davon an (001), was diese ablehnt (002). In der folgenden Gesprächspause nutzt Tatiana die Gelegenheit, einen vorher angeschnittenen Aspekt, ihre eigenen grauen Haare, nochmals ins Gespräch zu bringen. Dieser Redebeitrag ist zu Beginn nicht als Erzählung gerahmt, wird im weiteren Verlauf durch eine von Lars fremdinitiierte Reparatur jedoch dazu ausgebaut.

Tatiana, die als einzige noch nicht aufgegessen hat, führt in der Gesprächspause (003-007) eine Gabel mit Essen zum Mund. Allerdings kündigt sie gleichzeitig hörbar einen neuen Redebeitrag an und besetzt so dennoch den Slot des nächsten Sprechers (008, Abb. \#1). Sobald sie die Nahrung in den Mund geschoben hat, richtet sie sich auf und formuliert einen Nachtrag zum Thema ihrer grauen Haare (012-015). Im Transkript ist jedem Teilnehmer/jeder Teilnehmerin ein Zeichen zugeordnet, mit dessen Hilfe jeweils relevante sichtbare Handlungen annotiert werden (Tat: *, Lar: +, Mar: \%, Sim: \$, Bilder sind mit \# und laufender Bildzahl eingefügt).

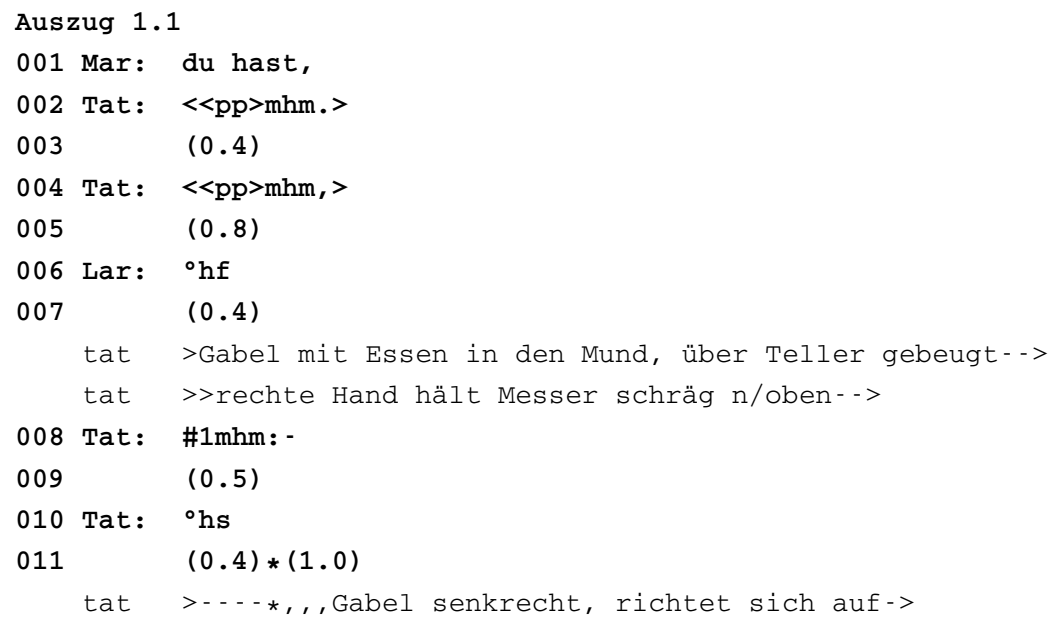

\footnotetext{
${ }^{3}$ https://franzoesistik.philhist.unibas.ch/fileadmin/user_upload/franzoesistik/mondada_multimodal_con ventions.pdf.
} 

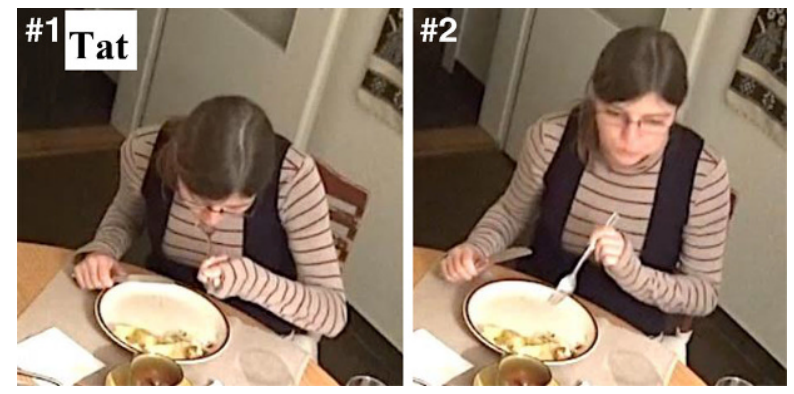

012 Tat: \#2ich will +ERNST *genommen werden.=

tat >..Blick vorn/SIM-*, , ,Blick n/unten->

tat >Essen im Mund -............. >017

lar >Blick vorn+...Blick TAT-.........

013 Tat: $=<<$ p $>$ deswegen $->$

$014 \quad($.

015 Tat: $<<$ plass ich [mir die graUen [haare.> ]

016 Mar: [.ts [jA das hast du]*schon [mal] gesAGT-

017 Tat: $\quad[<<p>m h m,>]$

tat $\quad$ *nickt, zwinkert>

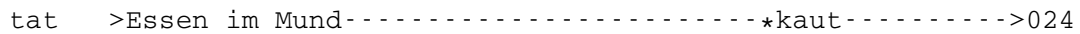

018 Mar: [hm;_hm, \%j] a* :,

019 Tat: [hf; hf, ]

mar >Blick Tat\%, ,

tat >nickt......*

020 Tat: hf\#3_\$hf*_h;\#4

tat $>$ MAR - - - *..SIM->

sim \$..Blick TAT->040

$021 \quad(0.2)$

022 Lar: zum \#5 Ernstgenommen: werden:;

023 deswegen trÄgst du die*:-=<<pp>öh;>*

tat $\quad \star$ nickt $-\ldots .->$

tat >Blick LAR-..............
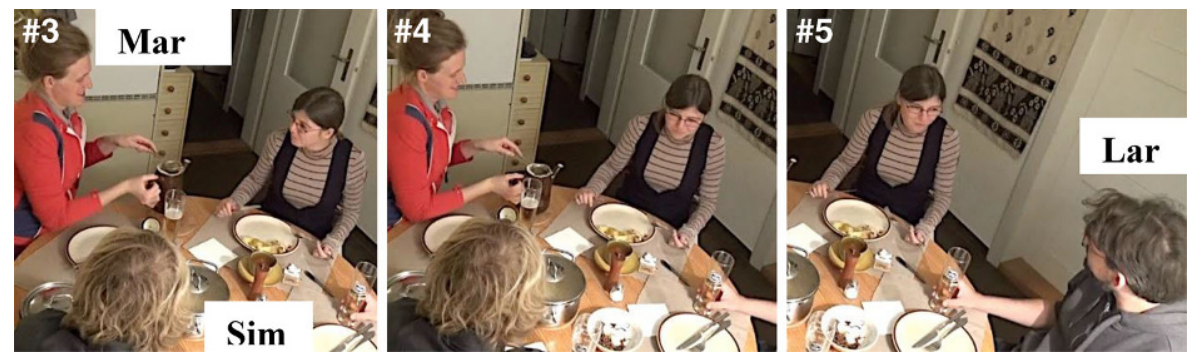
024

$*(0.3-\ldots . .-2) *(0.3)$

tat $*$ schliesst Augen $-*$. Blick LAR $->$

tat >nickt.........

tat >kaut-......-..schluckt Bissen herunter

025 Tat: [.ts grAUen] $*$ haare. $*=$

026 Lar: [okay- ]

tat $\quad$ nickt - *

027 Tat: $=\%<<$ acc $>$ des [wegen ] mach ich die nich WEG. $>+$

028 Lar: [ $+<<$ pp $>$ mhm- $>]$

mar $\%$....steht vom Tisch auf, holt Bier aus Kühlschrank-->

lar

029

030 Lar: $+*$ hm $-+*=$

lar +nickt $1 \mathrm{x}^{+}$

tat *schluckt*

$031 \mathrm{Sim}:=$ ja-

032 Lar: okay.=

033 Tat: $=* i-i c h+m U s s$ -

tat $*$..Blick SIM->038

lar >Teller--..Blick Tat->040

$034 \quad$ (.)

035 Tat: öh: $\mathrm{m} ;=$

036 Tat: =auch, \#6

$037 \quad(0.25)$

038 Tat: *HEUte noch *und IN deutsch*land;*\#7=

tat >-Blick SIM-*, Augen zu--.*..Blick LAR->040

tat $*$ Hände m/Besteck minimale Gesten-*

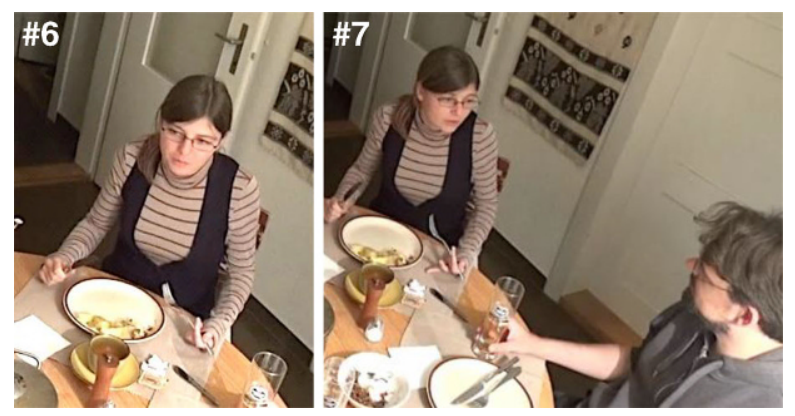

039 Tat: =es is mir wirklich-=

040 Tat: $=*$ im sOmmer is $\operatorname{mir}$ das passiert; $=i c h *$ wOllte-=

tat $\quad *$, , Blick $\mathrm{n} /$ unten -.................

Da Tatiana während ihres ersten längeren Redebeitrags (012) weiterhin Essen im Mund hat, das Besteck schräg aufgerichtet in den Händen hält (Abb. \#2) sowie relativ schnell wieder nach unten schaut (012), scheint sie zu diesem Zeitpunkt 
keinen längeren Beitrag zu projizieren, zumal Marie diese Aussage als zumindest ihr bereits bekannt markiert (016). Der Sequenzabschluss wird hier durch gemeinsames Lachen und Blickkontakt mit Marie hergestellt (018-020, Abb. \#3). Tatiana blickt daraufhin zu Simon (020, Abb. \#4), von dem keine Reaktion erfolgt, schließlich zu Lars (Abb. \#5), der eine Reparatur initiiert (022-023). Diese wird von Tatiana mit einem Nicken (023-024, 025) sowie, nach dem Herunterschlucken eines Teils ihres Bissens (024), einer verzögerten Ko-Konstruktion (025, Lerner 1996, 2004) und einer wiederholten Erklärung durchgeführt (027).

Obwohl die Adressaten Lars und Simon nur minimal antworten (028, 030-032) und Marie unterdessen vom Tisch aufgestanden ist (028), setzt Tatiana nun zu einem neuen Beitrag an (033-038). Dabei wandert ihr Blick von Simon (033-038, Abb. \#6), der sie seit Zeile 020 anschaut, zu Lars, mit dem sie ebenfalls gegenseitigen Blickkontakt etabliert (038, Abb. \#7, s. 033). Sobald sie die Aufmerksamkeit beider Rezipienten gesichert hat, schaut sie nach unten und beginnt die Erzählung eines beispielhaften Erlebnisses, das sie mit einer zeitlichen Einordnung einführt (040, »im sOmmer«). Interessanterweise legt sie ihr Besteck nicht aus den Händen, was eine baldige Wiederaufnahme des Essvorgangs projiziert (038, vgl. bodytorque, Schegloff 1998) und daher einen eher kurzen Beitrag, jedoch nicht notwendigerweise eine lange und als solche ausgebaute Narration. Vielmehr ist Tatianas Beitrag zunächst als eine Antwort auf Lars' Reparaturinitiierung zu verstehen, der ihre Aussage zu den grauen Haare accountable macht. Der folgende Ausbau des Erzählprozesses ist also sowohl auf der sequentiell-sprachlichen als auch auf der körperlich-visuellen Ebene von den Interagierenden nur schwach relevant gemacht.

\subsubsection{Ausgestaltung der Erzählung}

Während der längere Redebeitrag Tatianas ab Zeile 040 also primär als account formatiert ist, entwickelt sich dieser hin zur Erzählung einer Anekdote. Die kurze Rekonstruktion des Bierkauferlebnisses (Tatjana wird für zu jung gehalten, um Bier kaufen zu dürfen, 040-051, hier ausgelassen), die auf Tatianas Nutzen ihrer "grauen Haare im Alltag hinweist, wird durch minimale Bewertungen von Lars und Simon abgeschlossen $(052,055)$. Dass die eigentliche Erzählung erst jetzt folgt, kontextualisiert Tatiana in einer minimalen, körperlichen Vorbereitung: Während ihre Rezipienten antworten, streckt sie zunächst den kleinen Finger der linken Hand aus und schiebt ihn unter ihren Tellerrand (052), dann streckt sie den kleinen Finger ihrer rechten Hand aus und schiebt auch diesen unter den Teller (055, Abb. \#8). Zwar behält Tatiana weiterhin das Besteck in der Hand, jedoch bringt sie ihre Hände so in eine stabile Ruheposition, die bis zum möglichen Ende der Narration gehalten wird (076). Dies hat zur Folge, dass in der Ausgestaltung der nun folgenden Erzählung auf gestische Elemente weitgehend verzichtet wird. Stattdessen gestaltet die Erzählerin ihren Beitrag durch leichte Bewegungen des Oberkörpers und des Kopfes sowie hörbar-prosodische Ressourcen aus.

Auszug 1.2

052 Lar: <<creaky>oh* : ;

tat $>$ kl. Finger*...an/unter Tellerrand->076 


$$
(0.5) *(0.3)
$$

tat >LAR $-\star \ldots$. Blick SIM- - >

054 Tat: ${ }^{\circ} \mathrm{hhf}$

055 Sim:

$<<$ p $>$ kr $(\mathrm{h}) \mathrm{a} * \mathrm{ss}:->\# 8$

tat

*rHand kl. Finger ausgestreckt, an Teller/Tisch->076

056

$(0.2)$

057 Tat:

<<:-)>da hab ich* chrIstian HOLEN müssen; >*

tat

*Oberkörper/Kopfschütteln, richtet sich auf*

058 Tat: thh_ ${ }^{\circ} \mathrm{h}^{\circ} \mathrm{hi}$ ?

$059 \quad(0.7)$

060 Tat: [<<:-)>der in der nähe] war; >=

061 Mar: [\%hf_hf_hf ]

mar \%.lächelt--->066

062 Tat: $\left.={ }^{\circ} \mathrm{hf}<<:-\right)>$ und gesagt* $\operatorname{chr}(\mathrm{h})$ isti+(h) an>=hmf;_hmf,

tat >Blick SIM-.-.-.....n/unten, beugt Kopf vor->

lar >rHAnd an Bierglas...................... Bierglas zum Mund->

$063 \quad$ \#9 (0.3)

064 Tat: *<<:-)>ich wollt dir BIER \#10+kaufen;=aber ich+D(h) URfte

tat *...Kopf n/oben, Blick SIM n/vorn-........

mar >leert Bierdose in SIMs Glas-........-. >074

lar >schaut zu seinem Glas-........Blick TAT-....-

lar >hebt Bierglas zum Mund-..................trinkt>

$065 \mathrm{n}(\mathrm{h}) \mathrm{icht} ;>$

066

$$
(0.2--) \%
$$

mar

$>$ lächelt\%
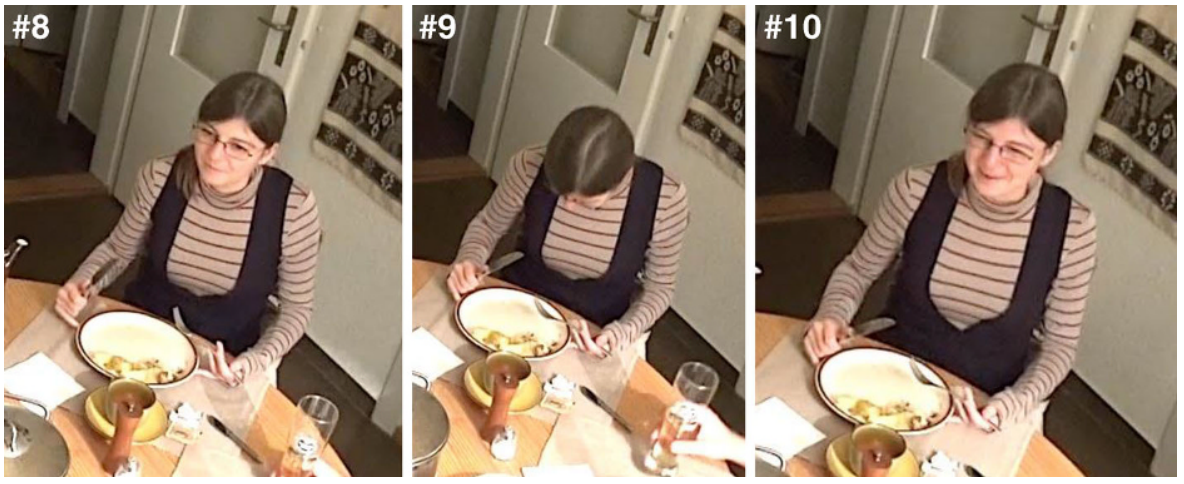

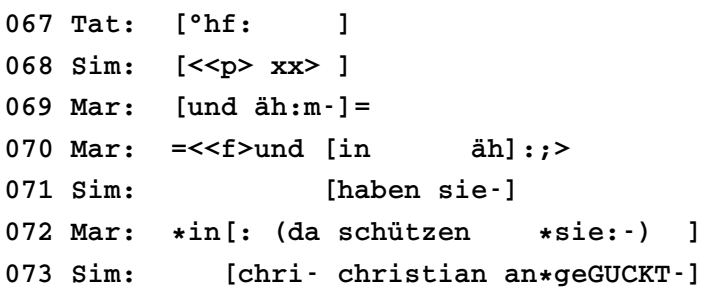




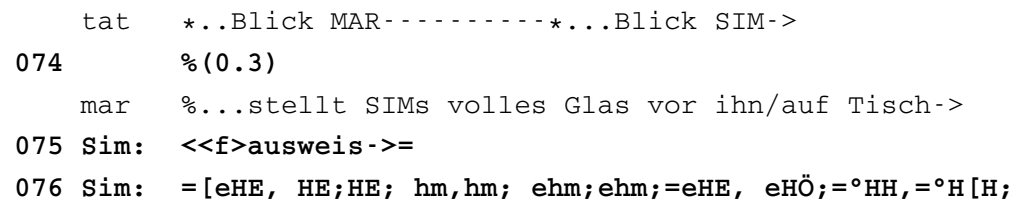

Der Einstieg in die Erzählung wird von Tatiana durch eine klar mit der vorherigen Rede kontrastierende Stimmqualität durchgeführt, da sie nun mit lächelnder Stimme spricht sowie ein höheres Tonregister wählt (057). Zudem mimt sie mit einer leichten, schüttelnden Bewegung des Oberkörpers und Kopfes ihre Empörung darüber, den Freund beim Bierkauf um Hilfe bitten zu müssen. Auf ihr vor dem Relativsatz eingefügtes Lachen (058-060) antwortet zunächst nur Marie (061). Im nächsten Teil der Erzählung, der Redewiedergabe, erhöht Tatiana daher die laughability ihres Beitrags: Sie fügt Lachpartikeln ein und beugt während und nach der Anrede lachend ihren Kopf nach vorne (062-063, Abb. \#9; vgl. Ford/Fox 2010). Als sie wieder hochschaut, weisen auch ihre nach oben gezogenen Mundwinkel sowie zusammengepressten Augen deutlich auf den Unterhaltungswert der Begebenheit hin (064, Abb. \#10), was schließlich nochmals durch das Hinzufügen von Lachpartikeln verstärkt wird (Ende 064, 065).

Auffällig ist hier, dass Tatianas Adressaten nur teilweise zur Verfügung stehen. Marie, die am frühesten antwortet (061), ist damit beschäftigt, am Tisch stehend Bier in Simons Glas zu gießen (bis 074). Sie setzt daher verspätet zu einem auch eher ernsthaft gerahmten Beitrag an, der jedoch unvollendet bleibt (069-070, 072). Lars, der bereits seit Beginn des Auszugs (001) sein Bierglas mit der rechten Hand umfasst, führt während Tatianas Ausführungen das Glas zum Mund (062) und beginnt, daraus zu trinken (064). Er antwortet daher nicht auf die simultan formulierte Klimax. Nur Simon, der von Tatiana vorwiegend angeschaut wird, formuliert eine imaginäre Weiterführung der erzählten Szene (071, 073, 075). An dieser Teilsequenz zeigt sich, wie parallel ausgeführte Aktivitäten der Teilnehmer zu erschwerten Ausgestaltungs- und Rezeptionsbedingungen einer Erzählung führen können.

\subsubsection{Ausstieg aus der Erzählung}

Es gibt zwei potentielle Stellen, an denen der Ausstieg aus der Erzählung möglich ist, welche jedoch durch die Expansion eines Rezipienten und dann durch die Erzählerin selbst verschoben werden. Obgleich Tatiana ihr Messer (das sie bisher ebenso wie die Gabel weiter in der Hand gehalten hat, Abb. \#11) in der rechten Hand wieder Richtung Teller kippt (079-080, Abb. \#12) und so das Ende ihrer Erzählung projiziert, werden ihre Essvorbereitungen erneut ausgesetzt. Lars, der in der Zwischenzeit sein Bierglas wieder abgesetzt hat, formuliert eine Expansion von Simons Witz (ab 078). Tatiana blickt daher zu ihm und legt ihre rechte Hand wieder auf dem Tisch ab (081, Abb. \#13).

Auszug 1.3

$076 \mathrm{Sim}:=\left[* \mathrm{eHE}, \mathrm{HE} ; \mathrm{HE} ; * \mathrm{hm}, \mathrm{hm} ; \# 11 \mathrm{ehm} ; \mathrm{ehm} ;=\mathrm{eHE}, * \mathrm{eHÖ} ;=\star^{\circ} \mathrm{HH},={ }^{\circ} \mathrm{H}[\mathrm{H}\right.$;

077 Tat: $[*$ hh 
078 Lar:

[oder -

tat *schliesst Augen*Kopf runter, lacht-.......Kopf hoch/SIM>

tat $>$ kl. Finger lks/rchts unter Tellerrand*,,, ,

$079 \mathrm{Sim}: \quad\left[*^{\circ} \mathrm{HI}\right.$,

080 Lar: [*oder $-\# 12$

tat $\quad$...rHand vom Tisch, kippt Messer, Essensvorbereitung->

081 Lar: *oder die <<:-)>standpauke=\#13

tat $*$. Blick LAR -......

tat $*,$, , legt rechte Hand mit Messer wieder auf Tisch ab->
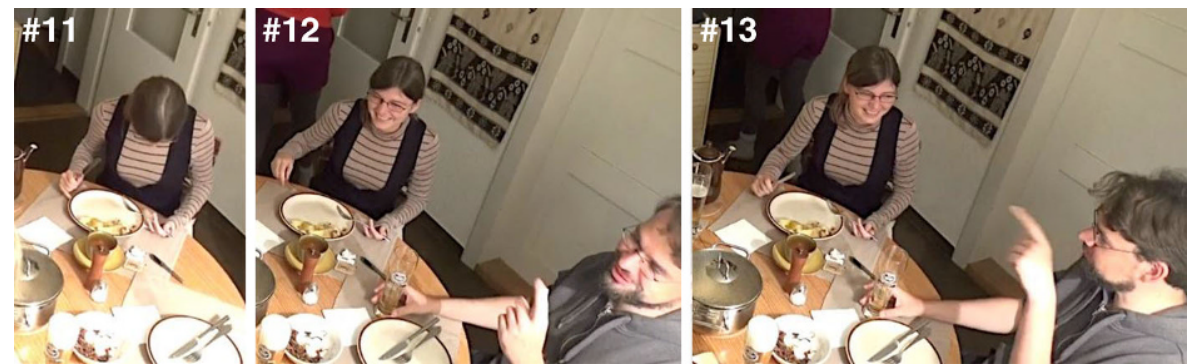

082 Lar: =+verboten \$fÜr MINder*jäh[rige $+[(\mathrm{zu} \# 14 *$ kaufen. $)>]=$

083 Tat:

$\left[{ }^{\circ} \mathrm{HI}, \mathrm{hm}\right.$;

084 Sim:

$\left[{ }^{\circ} \mathrm{HHhö} ;\right.$ he, $]=$

lar trHand Zeigefinger ppp , Schild'+, , , ,

lar >Blick n/vorn-..........................

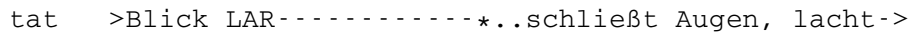

tat >lHand auf Tisch-..-*...ppp lZeigefinger $*,,,$, ,

sim \$...rHand zum Bierglas.......

085 Lar: $=[\mathrm{HE}, \mathrm{HM}$,

086 Sim: $=\left[j a(h) \_\right.$A $]$genau_(h) au= $(h) \$ a u f * j(h)$ eden [fall $(h) . \# 15=$

087 Tat:

$\left[{ }^{\circ} \mathrm{hh}\right.$

tat >hält Messer \& Gabel hoch-..-.*...zum Teller-.->

sim >rHand am Bierglas-..........hebt Bierglas an-->
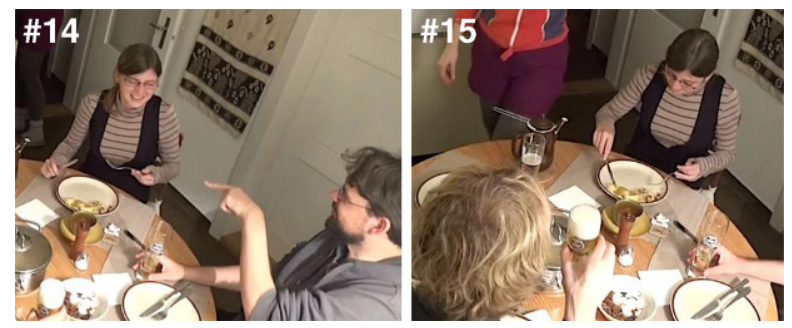
088 Sim:
$=e{ }^{\circ} \mathrm{HHE}=[\mathrm{he} ;$-he,
]
089 Lar:
$=[<<:-)>$ (und) $x$,
090 Tat:
[*wir hams - ] 


$$
\begin{aligned}
& \text { tat } \quad \text { *..hebt Kopf, Blick LAR }-> \\
& \text { tat } \quad \text { *..stellt Besteck schräg--> }
\end{aligned}
$$

091 Tat: <<:-)>(wie ga:nz;) ganz *dezent gemacht;>=
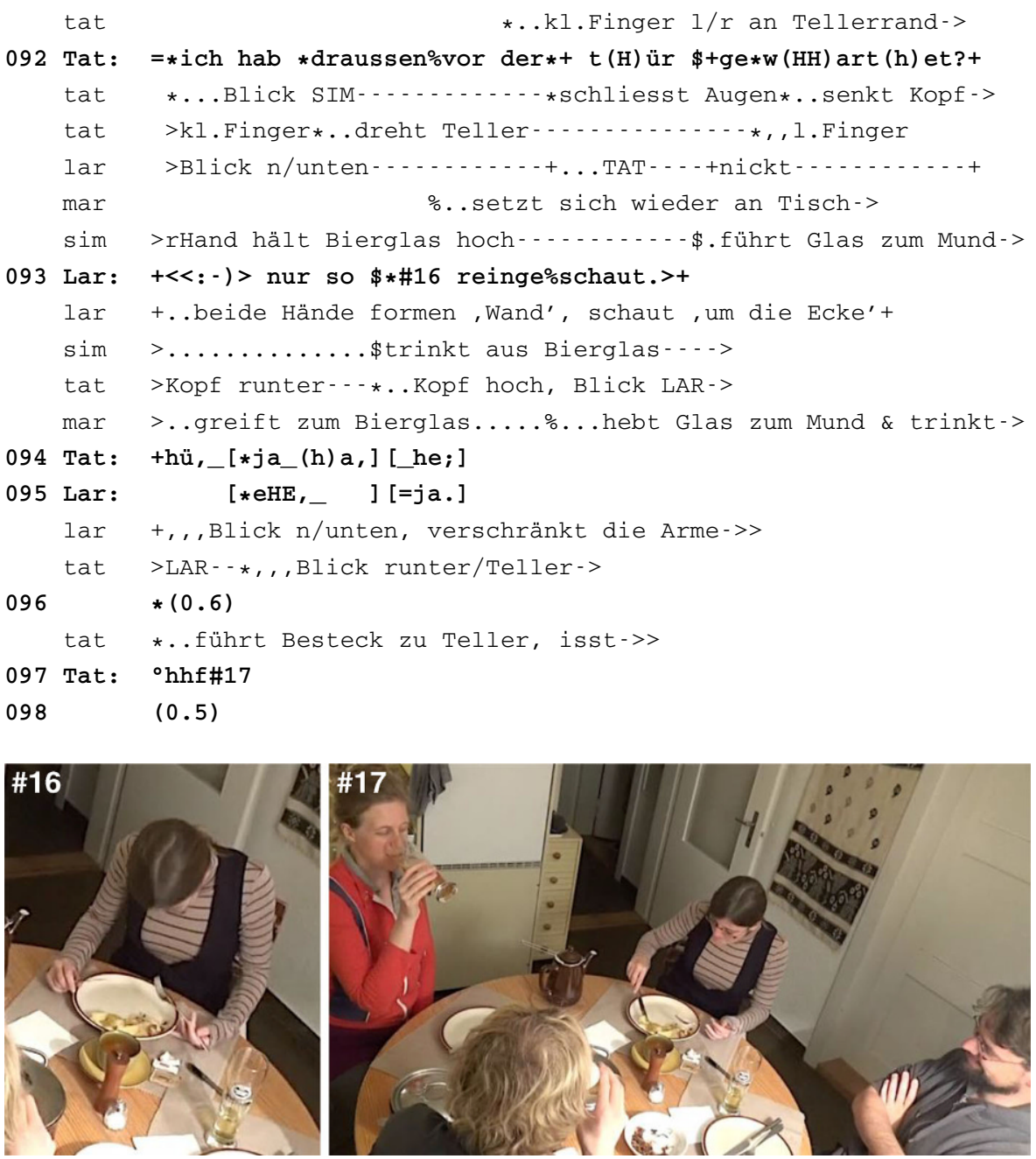

Lars, der bereits mit dem Essen fertig ist, nutzt nun die linke Hand, um gestisch auf ein imaginäres Schild mit dem von ihm formulierten Hinweis zu verweisen (vgl. Stukenbrock 2014 zur Deixis in einem imaginären Raum), indem er mit rhythmischen Zeigegesten von links nach rechts geht (082, Abb. \#12, \#13 und \#14) und so die »Standpauke« (081) inszeniert. Dies wird von Tatiana mit einem erneuten Lachdisplay (zusammengekniffene Augen, leises Lachen) sowie einer schnellen Zeigegeste mit dem linken Zeigefinger quittiert (083, Abb. \#14), sie nimmt hier also eher die Rolle einer Rezipientin ein. Simons von Lachpartikeln durchzogene Bewertung des Witzes (086) schließt diese Sequenz potentiell ab. Darauf weist Simons Griff 
zum mittlerweile von Marie aufgefüllten Bierglas hin, aber auch Tatianas erneute Orientierung zu ihrem Teller mit Blick und Besteck hin (085-087, Abb. \#15).

Tatiana unterbricht jedoch kurz darauf die von ihr projizierte Wiederaufnahme des Essens, da sie das Besteck erneut schräg aufstellt, ihren Blick zurück zu Lars wendet sowie einen neuen Redebeitrag beginnt (090), mit dem sie ihre zuvor erzählte Anekdote beendet und so das wahre Ende des Erlebnisses den beiden von Simon und Lars erdachten hinzufügt (091-092). Die erneute Position ihrer kleinen Finger am Tellerrand sowie das wiederholte Display aus Lachpartikeln, zusammengekniffenen Augen sowie nach vorn gebeugtem Kopf verdeutlich den Zusammenhang mit dem bereits von ihr Erzählten (Abb. \#16, vergleiche Abb. \#9 und \#11). Auch hier antwortet Lars erneut mit einem mimisch-gestischen re-enactment (vgl. Sidnell 2006), indem er mit beiden Händen eine imaginäre Wand formt und um diese herumschaut (093).

Die anderen Rezipienten sind bereits vorher sichtbar aus der gemeinsamen Erzählaktivität ausgestiegen: Marie, die sich nun wieder an den Tisch gesetzt hat (092), greift zu ihrem Bierglas und trinkt (093, vgl. Abb. \#17). Simon, der bereits während seiner eigenen Bewertung von Lars' erstem Witz (086) seine Hand zum Bierglas bewegt und dieses angehoben hat, führt es während Tatianas letztem Beitrag zum Mund (092) und beginnt bei Lars' Nachtrag, zu trinken (093, vgl. Abb. \#17). Während des letzten verebbenden Lachens wendet auch Lars seinen Blick von Tatiana ab und verschränkt seine Arme vor dem Oberkörper (094-095). Tatiana schließlich beendet als letzte sichtbar den vorherigen Aktivitätsrahmen, indem sie ihr Besteck erneut zum Teller führt und weiter isst (096). Abb. \#17 zeigt, dass sich alle vier Teilnehmer nun anderen Aktivitäten widmen bzw. durch ihre deutliche Orientierung weg von den anderen Teilnehmern keine imminenten Redebeiträge projizieren. Erst hier ist also die von Tatiana durchgeführte und durch Lars und Simon jeweils individuell weiterentwickelte Erzählung für alle sichtbar (gemacht) abgeschlossen.

\subsection{Beispiel 2: »Zitrone«}

Das folgende Beispiel aus einer Unterhaltung zwischen den FreundInnen Kim, Eva und Jan exemplifiziert einen Erzählprozess, der eine hohe Dichte an narrativ-gestalterischen Mitteln auf sprachlicher und körperlich-visueller Ebene aufweist und - anders als das erste Beispiel - nicht durch Multiaktivität geprägt ist. Zudem werden verschiedene narrative Teilsequenzen hier nicht individuell, sondern von allen gemeinsam bearbeitet.

\subsubsection{Einstieg in die Erzählung}

Die Interagierenden haben sich um einen WG-Küchentisch versammelt, trinken gemeinsam Bier und unterhalten sich über Kunden, die im Einzelhandel sehr wenig Verständnis dafür zeigen, wenn neue MitarbeiterInnen angelernt werden und sich Kassiervorgänge dadurch verzögern. Jans Erzählung schließt sich an eine szenische Darstellung Kims an, in der sie verbal, gestisch und prosodisch kontextualisiert, dass sie das Kassieren an Supermarktkassen als zu langwierig wahrnimmt (011-012, 015). Jan installiert sich hier als Ko-Erzähler, indem er den von Kim dargestellten 


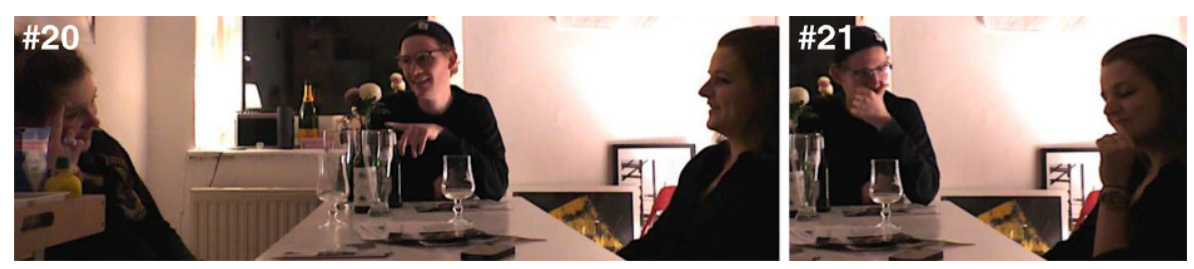

029 Eva: +die +fragen ja immer $\mathrm{NACH}$,

jan $+\ldots$...Blick Eva-......-.

jan >---+, , lZeigefinger, lHand z/Kinn->

030 und inzWIschen denk ich so-

$031 \quad$ \#21HM: : : ;

$032 \mathrm{Kim}: \mathrm{JA}$;

033 +LOHNT [sich; ]

034 Eva: [ hät]test du [gleich mal] (.) [ANfangen ]+soll [en; ]

$035 \mathrm{Kim}: \quad$ [ $(j \mathrm{~A}$ dann $] \mathrm{gu}[: t ;)]$

036 Jan: [hf: ] ] [ich HATte-]

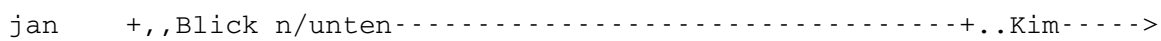

jan,+ , lHand an Bierflasche-.................................

037 Eva: [ (xxx) ]

038 Jan: [*\%ich\#22hatte +mal]+eine (.) äh kasSIErerin-\#23

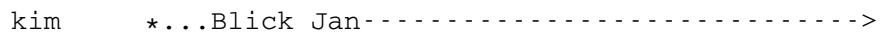

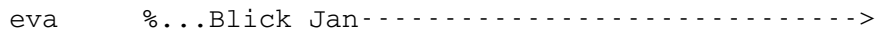

jan >lZeigefinger--+, , ,..spielt mit Kronkorken-->

jan >Blick Kim-......., , Blick n/unten/Tisch-.->
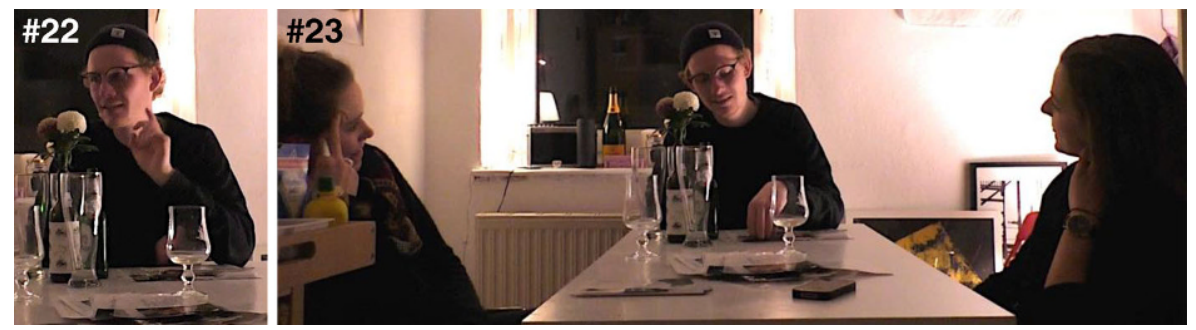

039

die war ein BISSchen-=

040

=+ich glaub die war_n bisschen AN+getrun+ken,

jan

+nickt leicht, hebt Kopf, Blick n/vornet, , unten->045

jan

041 Jan:

$042 \mathrm{Kim}$ :

043 Eva:

eva

eva

+ ...lächelt- ->

\begin{abstract}
un:d [die hat halt-]\% (.) \%die ganze zeit [verSUCHT-]
\end{abstract} [pf: he, ]

[he; hm, ]

044 Eva: [th hi; ] [\%hi, ]

045 Jan: [+ne (-) ] [+ne ] ziTROne zu bongen; = 


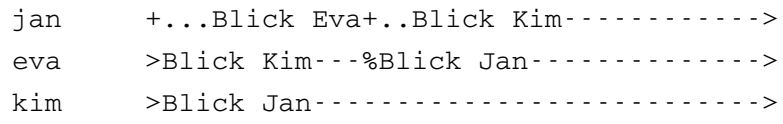

Jan projiziert eine Erzählung durch eine typische Einleitungsformel (»ich HATte (mal)«, 026, 028). Durch das Heben und Schütteln seines Zeigefingers (vgl. Bohle 2007, S. 198) sowie seinen Blick zu Kim kontextualisiert er einen thematischen Anschluss an ihren Beitrag und instanziiert sie als primäre Adressatin (Abb. \#19). Da sein Einsatz aber simultan zu Evas Kommentar zu ihrer Beteiligung an dem Bonuspunktsystem $(025,027)$ erfolgt - die sich sichtbar an Kim adressiert (Abb. \#18) -, wiederholt er bei den nächsten redeübergaberelevanten Stellen die narrationseinleitende Formel (028, vgl. Schegloff 1987). Dabei tippt er rhythmisch mit dem Zeigefinger auf die Bierflasche vor sich und schaut erneut zu Kim, die sich jedoch nun sichtbar als Rezipientin für Eva positioniert hat (Abb. \#20).

Daraufhin suspendiert Jan seine Erzählung, was er körperlich dadurch anzeigt, dass er beginnt, seine »Konkurrentin« Eva zu beobachten (029-033). Zeitgleich berührt er mit seiner linken Hand sein Kinn (029-033, Abb. \#21), dann die Bierflasche (033, vgl. Oloff 2013). Erst in Antizipation von Evas Kommentarende (034) projiziert er in Überlappung mit der gleichen Formel »ich HATte-/ich hatte mal« und mit ähnlicher Zeigefingergeste zum dritten Mal eine Folgeerzählung, wobei er sich erneut zu Kim wendet (036, 038, Abb. \#22). Während dieses dritten Einstiegs wenden ihm beide Teilnehmerinnen den Blick zu. Sobald sich Jan die Aufmerksamkeit Kims sowie den floor gesichert hat, blickt er auf den Tisch und baut seine Erzählung aus (038, Abb. \#23). Der von den Rezipientinnen abgewandte Blick zeigt auch die dem Erlebten vorangestellte Einführung der Hauptakteurin an, einer angetrunkenen Kassiererin (039-040, vgl. Sidnell 2006, S. 378).

Der Ausschnitt zeigt somit einen Prozess auf, in dem sich ein Sprecher aus der Beteiligungsrolle eines Ko-Erzählers heraus selbst als Erzähler einer Folgenarration installiert. In einem Kontext, in dem Eva und Jan um die sequenzielle Anschlussposition nach Kims Ersterzählung konkurrieren, erfolgt die Projektion von Jans Folgeerzählung nicht allein durch sprachliche Mittel, sondern auch durch spezifische erzählankündigende Gesten.

\subsubsection{Ausgestaltung der Erzählung}

Eine gestische Nachstellung, ein re-enactment des Scanvorgangs der Zitrone (s. 041, 045), wird erst dann realisiert, nachdem sich Jan der Aufmerksamkeit (zumindest) seiner Hauptrezipientin Kim und seiner Rolle als primärer Sprecher versichert hat (vgl. Sidnell 2006; Thompson/Suzuki 2014). Jan hebt dazu seine rechte Hand auf Kinnhöhe an, formt die Hand dabei so, dass sie das Halten eines imaginären Objekts, in diesem Fall der Zitrone, kontextualisiert, und zieht diese dann seitlich hin und her (046, Abb. \#24, \#25 und \#26). Im Folgenden beteiligen sich beide Zuhörerinnen durch responsives, verkörpertes Lachen, die Kommentierung des Erlebnisses sowie alternative gestische Ausgestaltungen an der Erzählung. 
Auszug 2.2

046

$047 \mathrm{Kim}$

048 Eva:

jan jan
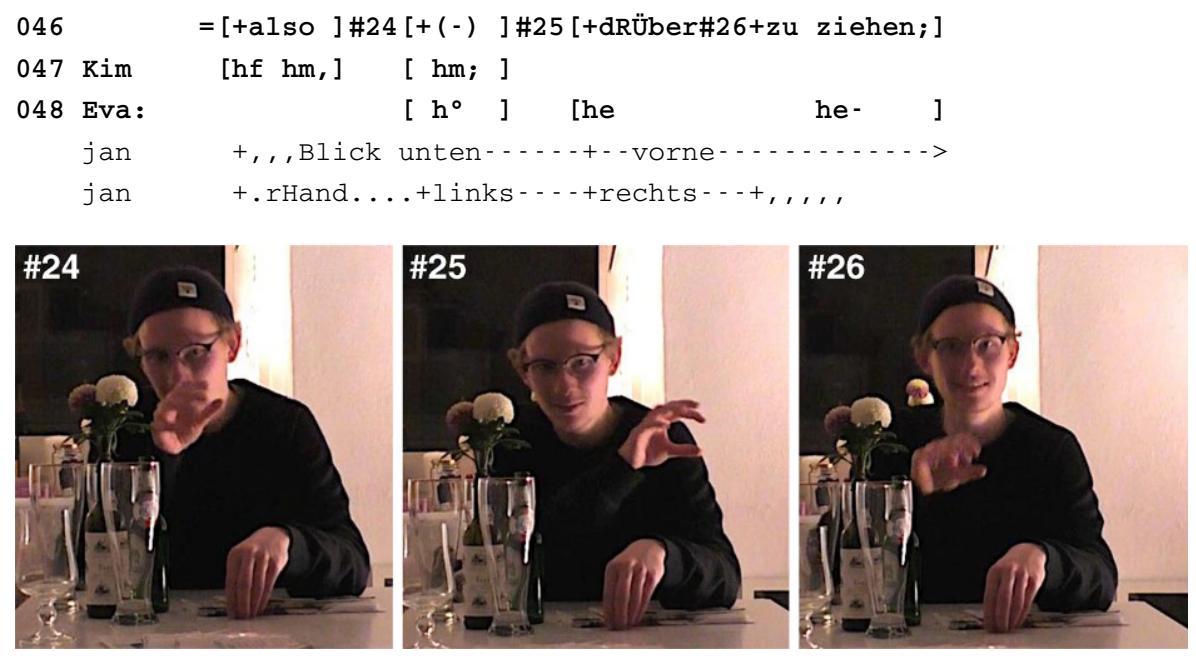
049 Jan: +und es hat +nicht funktio[NIERT; ]
$050 \mathrm{Kim}$ : [he - ]
051 Eva:
$[<<$ lachend $>$ das] + klingt so +GEIL; $>$

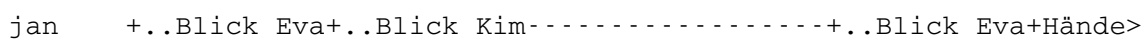
052 Kim: +\#27ha [hahahaha-
]
053 Jan: [und dann+(.) hat sie+\#28es da die ganze] zeit+da\#29
jan $\quad+\ldots$ hoch/links+, , rHand unter Tisch-->

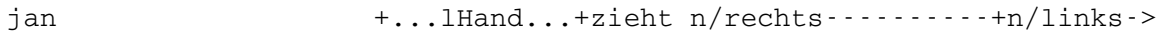
jan >Blick Hände, n/vorne->
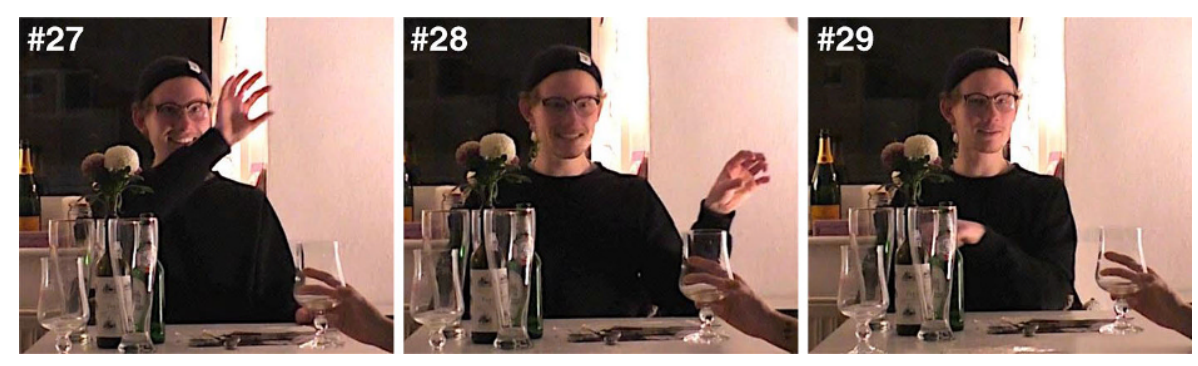
054 Jan: DRÜber ge [zog+en, ]
055 Eva:
056 Jan:
jan
jan
057
058
059 Eva:
[ ${ }^{\circ} \mathrm{h}$ he ] he [hehe- ]
[und+das hat] das PIEPT ja immer-
>links-...-. +rechts-......rHand openpalm up-...-.

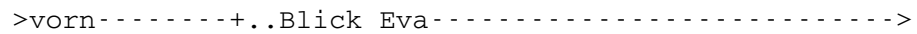
wenn das $\mathrm{NICH}-$
wenn das + nich (.) funktio[\%NI+ERT; ]
$[\% \mathrm{JA}+: ;]$



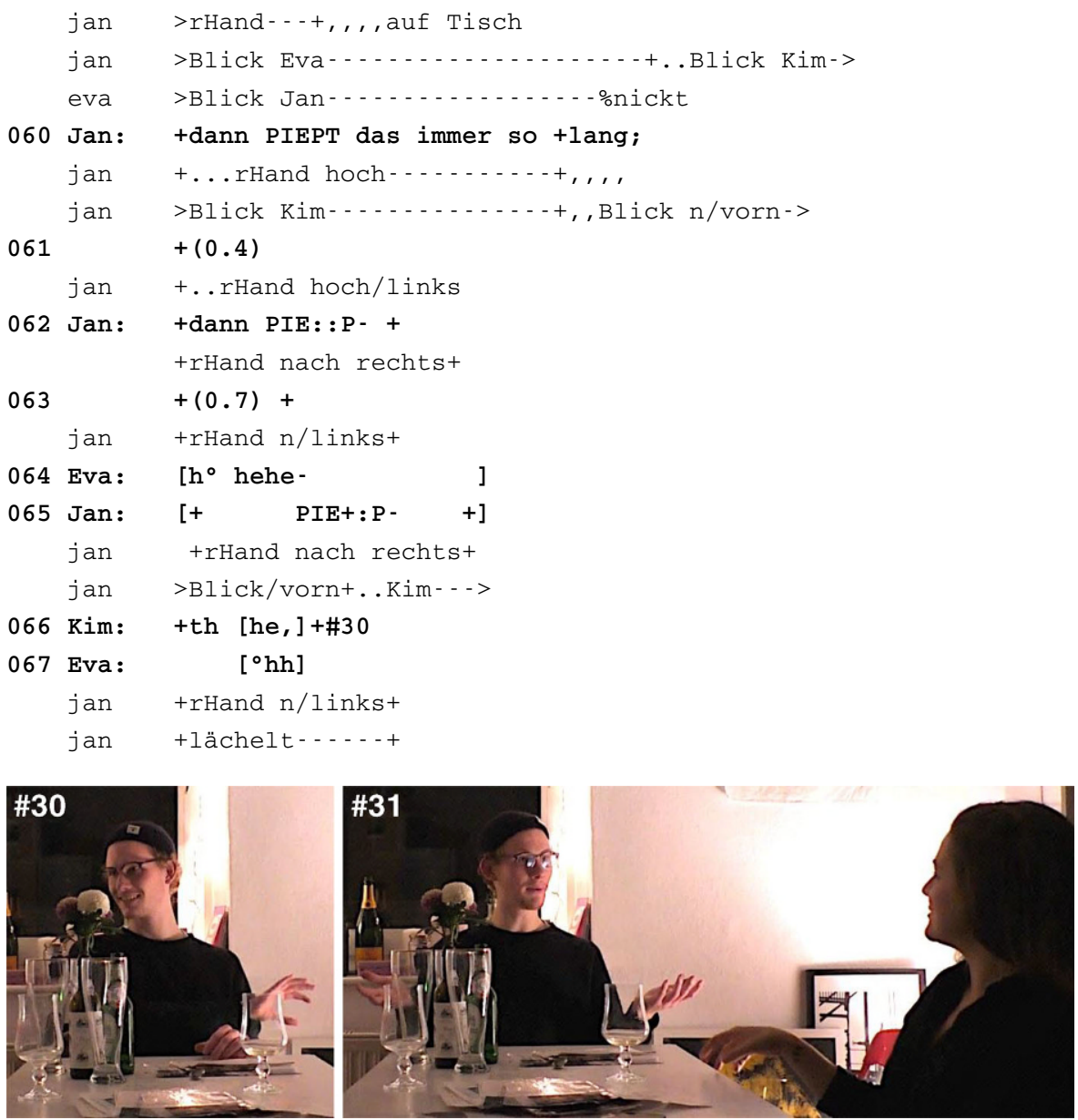

068

+das [+hat sie]

069 Eva:

[HÄ: - ]

jan

+...Hände open palm up zur Seite-->

jan

>Kim--....Blick Eva-.......... >

070 Eva:

+aber\%bei [ gemüs+e *musst du \%das

[nur \#32DRAUF] legen-

071 Jan:

[VIER fünf+\#31mal hintereinan[+der gemacht,]

072 Kim:

[*also*ich] muss das $x x-$

jan

+schüttelt Kopf--- -

jan

>palms up-...-.+, , Hände fallen a/Tisch+

$\mathrm{kim}$

>Blick Jan-..........Blick Eva-........Blick Jan-.->

$\mathrm{kim}$

*..lHand palm/Tisch-->

eva

$\%$..rHand a/Tisch

eva

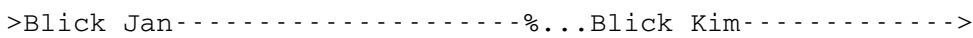




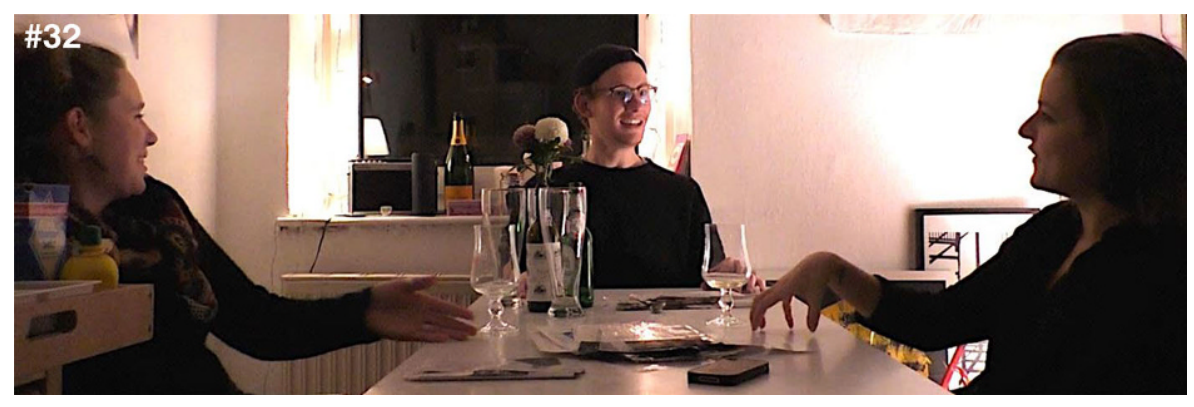

\begin{tabular}{|c|c|c|}
\hline 073 & Eva: & [\%und so WIEgen \\
\hline 074 & Jan : & [+das that sie aber nicht\#3 \\
\hline & eva & \%rHand Zeigefinger tippt \\
\hline & jan & $+\ldots l e h n t$ sich vor, Schul \\
\hline & jan & +Kopfschütteln-...- - \\
\hline & Eva : & $\% * h^{\circ} \# 34 h e \%$ he $\left[\right.$ he $-{ }^{\circ} \mathrm{hh}$ \\
\hline & Kim: & [ha ha, ha;\#35 \\
\hline & eva & \%wirft Kopf zurück\%, , \\
\hline & $\mathrm{kim}$ & *beugt sich lachend vor-> \\
\hline
\end{tabular}
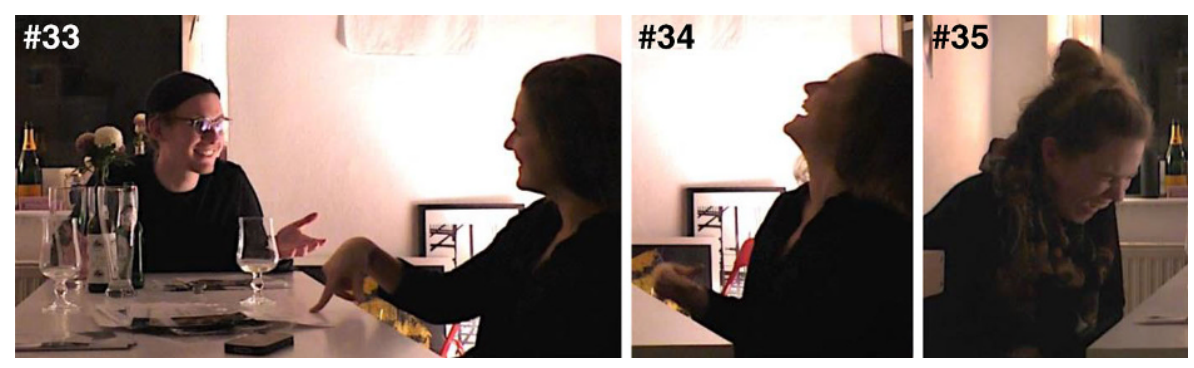

077 Jan: [+sie hat nicht verSTANden- dass sie das]+[WIEgen muss-]

$\begin{aligned} 078 \mathrm{Kim}: & \text { [*hehehahaha; }] \text { [haha, ] } \\ \mathrm{kim} & \text { >*..lehnt sich lachend zurück-......... }\end{aligned}$

jan $\quad+\ldots$ Hände open palm up zur Seite/Tisch--+, , ,

$079 \mathrm{Kim}$ : [haha, haha; ]

080 Jan: [also dass sie das irgendwie EINtippen muss;]

081 Jan: [sie hat f:- ] [sie hat geDACHT-] [das WÄR halt-]

$082 \mathrm{Kim}$ : [haha ] [hahaha ]

083 Eva: [ [ ${ }^{\circ} \mathrm{hhh}$ ] [he, <<h>HEI]:SS->

$084 \mathrm{Kim}$ : aha, [haha [haha [hahaha;] [ ${ }^{\circ} \mathrm{hh}$

085 Jan: [(wirklich-)] [(irgendwie)+(-) [.ts+

086 Eva: [ehe: [hehehe-]

jan +Kopfschütteln+

087 Jan: [+ (und dann-] 
$088 \mathrm{Kim}:$

jan

[ja KLAR - ]

Nachdem durch Lachen (047, 048, 050, 052) und Bewertung (051) eine Bestätigung der Erzählwürdigkeit erfolgt ist, baut Jan die narrativ-szenische Rekonstruktion weiter aus. Nicht nur sein »und dann« projiziert eine Fortführung der Erzählung; gleichzeitig hebt er erneut seine rechte Hand - diesmal auf Augenhöhe -, wodurch er eine Fortführung des reenactments ankündigt (053, Abb. \#27). Im weiteren Verlauf der Einheit führt er die rechte Hand unter den Tisch und wechselt zu einer gestischen Darstellung des Scanvorgangs auf die linke Hand, die er zweimal von rechts nach links zieht (053, Abb. \#28 und \#29). Mit seinem zumeist nach vorne bzw. ins Leere gerichteten Blick verkörpert Jan hierbei die erzählte Welt (vgl. Sidnell 2006; Ehmer 2011, S. 61), die Kassiererin sowie deren automatisiertes, unreflektiertes Handeln.

Gegen Ende seines Enaktierens schaut Jan zu Kim und beginnt zu lächeln, was eine Reaktion relevant macht (065-067, Abb. \#30). An dieser Stelle nun begnügen sich beide Zuhörerinnen nicht mit einem responsiven Lachen (064, 066-067), sondern nutzen diesen von Jan kreierten Slot als Möglichkeit, sich an der Erzählung zu beteiligen. So expliziert Eva nun das von Jan durch seine Handhaltung und das Kopfschütteln dargestellte Unverständnis über die Kassiererin (s. Abb. \#31), indem sie das eigentlich erwartbare Kassierprozedere ausformuliert (069-070, 073), in Überlappung und möglicher Redewiedergabe kommentiert auch Kim das soeben Gehörte (072). Auch gestisch nutzen beide diese intra-narrative Beteiligungsmöglichkeit, Kim mit einer kurzen Zeigegeste zum Tisch bzw. zum imaginären Kassenband, Eva mit einer zunächst ein Objekt darstellenden rechten Hand auf dem Tisch, dann mit einer tippenden Zeigefingergeste, die sich auf den Vorgang des Abwiegens und Eingebens bezieht (Abb. \#32 und \#33). Simultan dazu expliziert Jan das Nichtverstehen der Kassiererin dieser üblichen Vorgehensweise (074), was er auch körperlich durch Kopfschütteln, Schulterzucken, erhobene Augenbrauen, Vorlehnen sowie Lächeln zum Ausdruck bringt (Abb. \#33).

Erst nachdem die Pointe dergestalt von den drei Teilnehmern simultan und mehrfach verbal sowie gestisch ausgebaut wurde, wird diese zusammen goutiert. Kim und Eva veranschaulichen ihr Amüsement durch heraufgestuftes, klar verkörpertes Lachen (vgl. Ford/Fox 2010) und markieren somit die narrative Klimax der Erzählung (vgl. Selting 2017): Eva wirft den Kopf in den Nacken (075, Abb. \#34), Kim beugt sich lachend und mit geschlossenen Augen vor (076, Abb. \#35). Jan hingegen positioniert sich durch Wiederholung des Problems sowie einer ähnlichen Körperhaltung erneut als Erzähler, der durch das Lachen beider Zuhörerinnen begleitet wird (077-086). Dies erfolgt auch dadurch, dass er nun den von Eva erwähnten Vorgang nochmals verbal bearbeitet (080-081) und schließlich mit einer stärker bewertenden Inszenierung abschließt: Die multimodale Annotation zeigt, dass er sich vorlehnt und aus übertriebener Nähe die imaginäre Zitrone »begutachtet«(087).

Die Erzählung ist hier jedoch noch nicht abgeschlossen, da die Teilnehmer im weiteren Verlauf nochmals gemeinsam den Ausgangspunkt der Erzählung, das Scannen der Zitrone, bearbeiten (088-101, hier ausgelassen). Hierbei enaktieren erst Kim, dann Eva gestisch und prosodisch den Scanvorgang, bevor Jan abermals eine Kurzfassung seiner zweimaligen Inszenierung darbietet. Nach der gemeinsamen Ausar- 
beitung und dem Goutieren der Pointe kehren die Teilnehmer zum Ausgangspunkt der Erzählung zurück, im Sinne einer narrativen Reprise oder Nachverbrennung (vgl. Schwitalla 2006). Auch diese erfolgt nicht durch den eigentlichen Erzähler allein, sondern wird reihum von allen Anwesenden bearbeitet, alle drei InteraktionsteilnehmerInnen sind daher beständig als story consociates (vgl. Lerner 2002) an dem Erzählprozess beteiligt. In der vorliegenden Erzählsequenz, in der die InteraktionspartnerInnen anders als in dem ersten Beispiel keinen weiteren Aktivitäten nachgehen, können also sowohl der primäre Erzähler als auch die Rezipientinnen eine große Bandbreite an Verfahren des szenischen re-enactments der rekonstruierten Handlung nutzbar machen.

\subsubsection{Ausstieg aus der Erzählung}

Während sich in dem vorherigen Ausschnitt zahlreiche Darstellungsverfahren finden, mit denen alle Interaktionsbeteiligten eine narrative Klimax signalisieren, kommt es in der Folge zu einer ausgedehnteren narrativen Reprise, die sich in ihrer multimodalen Ausgestaltung von der vorherigen Erzählsequenz absetzt. Die Interagierenden stellen so ebenfalls gemeinsam einen Ausstieg aus der Praktik des Erzählens her.

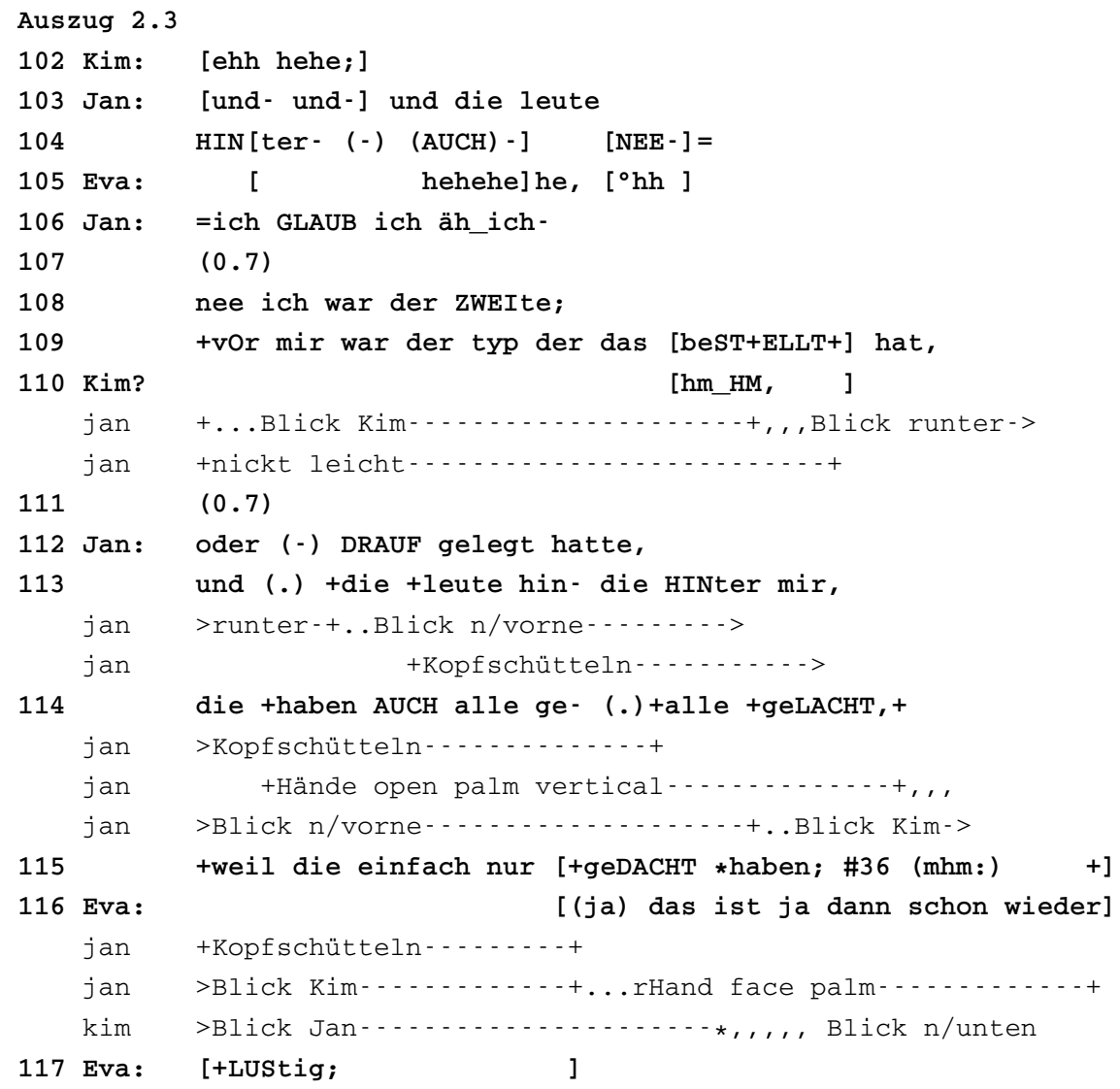


$118 \mathrm{Kim}: \quad[*<<$ p $>$ oh mein GOTT ey; $>]$

jan $\quad+$. Blick Eva-...... -

kim $\quad$....lHand vor Mund--->

119 Eva: \%also [weil \#37*SO ne] fehler kannst ja auch\%drüber 1Achen;

120 Jan? $\quad[<<p>$ ey das $\mathrm{xxx}>]$

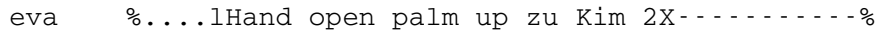

kim >Blick n/vorne-*...Blick Eva-..........
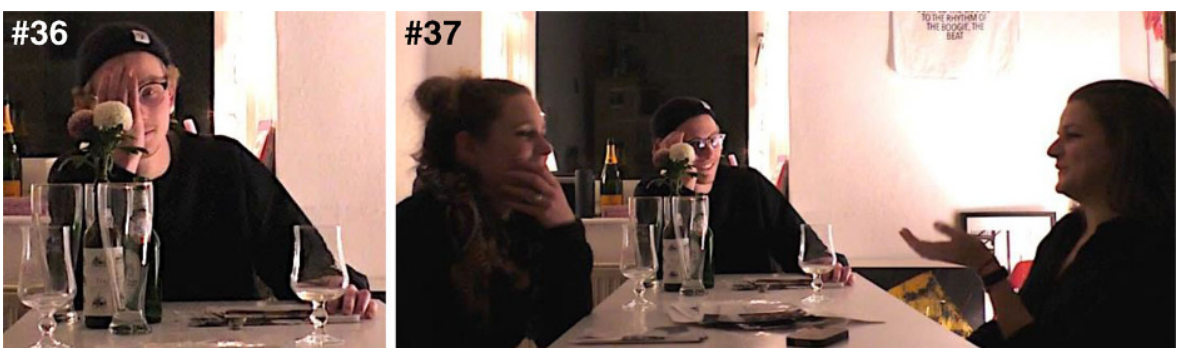

121 Eva: $\mathbf{x}[\mathrm{x}][\mathrm{xx}]$

122 Kim: [JA.]

123 Jan: [JA: ; ]

124 aber es WAR halt (mega) (.) ich-

125 und sie hat das überhaupt nicht verSTANden; +

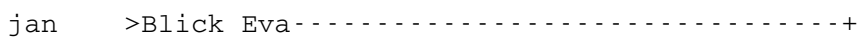

126 Eva: +JA $[:$,

$127 \mathrm{Kim}: \quad[\mathrm{hf}] * \mathrm{hf}$

jan +Blick n/unten-->136

kim >Jan---*, Blick n/unten-->132

$128+(0.6)$

jan +.greift nach Kronkorken a/Tisch, sammelt ein->>

129 Eva: hf hf [x hh

130 Jan: [dann so (.) HÄ,

warum $\uparrow$ KLAPPT das denn jetzt gAr nicht hier;

132

also (.) sonst hat das 个immer ge*KLAPPT;

kim >Blick n/unten-..............................

133 Eva: hehe,

$134 \quad(0.4)$

$135 \quad<<\mathrm{p}>\mathrm{OA},>$

136 Kim: *hier\#38 sollen+ wir *noch mal son: äh teil AUFmachen?=

kim *.zeigt z/Kühlschrank*,, , ,

jan >Blick n/unten-+...Blick Kim-->> 


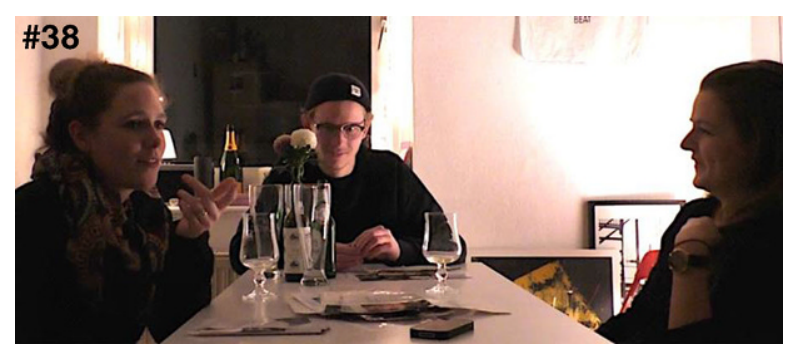

Der Chronologie des Erlebten folgend verweist Jan nun auf die Reaktionen anderer Supermarktkunden auf den Fehler der Kassiererin. Hierbei rekonstruiert er mit einer mit dem vorherigen Tonfall klar kontrastierenden Ernsthaftigkeit die genaue räumliche Situation an der Kasse (103-112). Die Erwähnung der Reaktion der anderen Kunden, Lachen (114), nutzt Jan dazu, nochmals bereits vorher von ihm verwendete, verkörperte Ressourcen einzusetzen und so erneut eine Bewertung der Kassiererin vorzunehmen (Kopfschütteln, ausgebreitete Arme, 113-114). Zusätzlich legt er sich schließlich die rechte Hand vor das Gesicht (facepalm) und komplettiert somit seinen syntaktisch unvollständigen Redebeitrag (115, Abb. \#36; vgl. Olsher 2004). Der Abschluss seiner Erzählung wird - neben dem von Eva und Kim abgewandten Blick - ebenfalls durch eine nun eher zusammengesackte Haltung und den an den Tisch gerückten Oberkörper verdeutlicht (115-116, Abb. \#36).

Simultan zu dieser abschließenden Bewertung formulieren dann auch Kim und Eva jeweils einen verbalen Abschluss der Erzählung. Kim vollzieht dies durch eine verbal und sichtbar alignierte Handlung (118, Abb. \#37, linke Hand vor dem Mund), während der sie ihren Blick bereits von Jan abgewendet hat. Wie der dann folgende Blickrichtungswechsel von ihr und Jan zu Eva zeigt (118-119, Abb. \#37), hat Jan hier seine Position als Erzähler eindeutig aufgegeben, somit ist der Übergang zur erneuten Zug-um-Zug-Verhandlung des Rederechts erfolgt. Während bereits Kims Bewertung einen möglichen Bezug zu dem Gesamtrahmen der Erzählsequenz herstellte (»Fehler neuer MitarbeiterInnen«), stellt Eva durch Redebeitrag, Blick und Geste zu Kim einen noch deutlicheren Bezug zu deren erster Narration her (116-117, 119, Abb. \#37).

In einer finalen, allein gestalteten Expansion (die eine weitere Möglichkeit für Eva bietet, eine expliziter auf die Erzählung bezogene, affiliativere Antwort zu geben, vgl. Jefferson 1978) entwickelt Jan ein letztes Mal das Motiv des Nicht-Verstehens (124-125, 130-132). Obgleich er nochmals die bereits vorher verwendeten körperlichen Ressourcen einsetzt (zusätzlich mit Redewiedergabe der Kassiererin, 130-132), wendet er kurz danach seinen Blick von Eva ab (Ende 125). Der Abschluss der Erzählung wird auch durch die Abwesenheit einer ausgebauten bzw. affiliativen Antwort Evas deutlich. Diese beschränkt sich auf minimales »ja« (126) sowie ein kurzes, leises Lachen mit leiser, evaluierender Interjektion (133, 135). Die Reaktionen fallen in dieser Phase - im Gegensatz zu dem lauten und körperlich inszenierten Lachen in der Kernphase der Narration - also eher verhalten aus. Auch bei Jan weist die prosodische Ausgestaltung der Redewiedergabe zwar noch Merkmale der Inszeniertheit auf, jedoch ist sein Blick während und auch nach der Redewiedergabe auf seine Hände ausgerichtet, die zeitgleich mit Kronkorken spie- 
len (ab 128, s. Abb. \#38), denen jedoch kein Objektstatus in der erzählten Welt zugeordnet wird. Die Interagierenden realisieren diese Sequenz durch diese Verfahren insgesamt als eine narrative Nachverbrennung, die einen Ausstieg aus der Erzählung ermöglicht. Dieser wird schließlich explizit durch Kim vollzogen, die, mit ihrem Blick von Jan weg nach vorne gerichtet, vorschlägt, eine weitere Flasche Bier zu öffnen (s. Zeigegeste, 136, Abb. \#38). Als Eva sich schließlich von ihrem Stuhl erhebt, wird der Teilnehmerrahmen (Goodwin/Goodwin 2004) der Erzählung endgültig auch räumlich-visuell aufgebrochen.

\subsection{Diskussion}

Während der erste Ausschnitt »Ausweis« veranschaulicht, wie die Praktik des Erzählens schrittweise und unter Zusammenarbeit aller Beteiligten vom vorherigen sequenziellen Kontext abgegrenzt wird, zeigt der zweite Ausschnitt »Zitrone« einen kompetitiven Einstieg in eine von Beginn an klar als Erzählung und als second story gerahmte Handlung. Auch wenn es sich in beiden Fällen um Tischgespräche handelt, divergiert die Ausgestaltung deutlich in Hinblick auf eventuell parallel laufende, körperliche Handlungen (Beispiel 1) oder deren Abwesenheit (Beispiel 2). Die Bedingung der Multiaktivität hat also erkennbare Konsequenzen für die nutzbaren Verfahren zur multimodalen Ausgestaltung des Erzählens. Die Situiertheit in einer face-to-face-Situation und damit die Potenzialität kinetisch-visueller Praktiken erweist sich gerade im zweiten Ausschnitt als besonders sichtbar: Der kompetitive Erzähleinstieg kann durch spezifisch ankündigende Gesten umgesetzt werden, der szenische Ausbau bzw. ein reenactment des rekonstruierten Erlebnisses wird ebenfalls stark über visuelle Ressourcen realisiert, wobei sich alle drei TeilnehmerInnen nacheinander vor allem gestischer Ressourcen bedienen. In beiden Fällen wird die Erzählung nicht durch einen einzelnen Redebeitrag, sondern schrittweise beendet, jedoch kann dies stärker mit der Wiederaufnahme praktischer Aktivitäten verbunden sein (Beispiel 1) oder aber durch einen gemeinsam konstruierten, sicht- und hörbaren Ausstieg aus dem Erzählrahmen stattfinden (Beispiel 2). Der Ausstieg aus dem Erzählprozess wird durch ein schrittweises Zurücknehmen der gemeinsamen Orientierung sowie der verbalen, vokalen und körperlichen Ressourcen kontextualisiert.

\section{Fazit: Der Alltag des Erzählens}

Erzählen ist eine zentrale Praktik im kommunikativen Alltagshandeln: Sie ist nicht nur häufiger Bestandteil von Alltagsinteraktionen, sondern sie konstituiert Alltag mit. Ihre Verwobenheit mit unterschiedlichsten kontextuellen Bedingungen bildet den analytischen Ausgangspunkt einer interaktionalen Narratologie. Ein zentraler und bislang nur in Ansätzen beachteter Aspekt ist die Situierung des Erzählprozesses in der körperlichen Ko-Präsenz. Diese bedingt, dass neben verbalen und vokalen auch visuelle und räumlich-materielle Ressourcen zur Einleitung, Ausgestaltung und Ausleitung von Erzählprozessen zur Verfügung stehen. Unsere Analysen zeigen zudem, dass Erzählungen nicht immer eindeutig aus einem bisherigen Handlungskontext ausgegliedert werden können: Wenn SprecherInnen im Alltag erzählen, bedeutet 
dies auch, dass die Erzählprozesse häufig in alltagspraktisches Handeln eingebunden sind. In diesem Sinne ist Multiaktivität eine wichtige Rahmenbedingung für die Ausgestaltung von Erzählungen, auch wenn sie bislang meist nur in institutionellen, deutlich empraktischen oder mobilen Zusammenhängen untersucht wurde (vgl. Keevallik 2013; Mondada 2014b; Nevile 2007). Betrachtet man jedoch alltägliche, nicht-mobile Multiaktivität (wie das Essen oder das Einschenken eines Getränks), so wird deutlich, dass auch alltägliche, routinierte Tätigkeiten stets mit sozialer Interaktion koordiniert werden. Wie unsere Beispiele kontrastiv aufzeigen, hat dies Auswirkungen auf die Art, wie TeilnehmerInnen erzählen (können): Gleichzeitige manuelle Tätigkeiten erschweren die gestische Ausgestaltung der Erzählung, die Koordination der Rezipienten mit der sequentiellen Struktur der Erzählung sowie die Aufrechterhaltung eines festen Teilnehmerrahmens (Bsp. 1). Sind alle Interaktanten hingegen ausschließlich auf das Gespräch fokussiert, setzen sie verstärkt Körperbewegungen beim Erzählen und Antworten darauf ein. Die Koordination mit den Rezipienten sowie die Kontrastierung von erzählter Welt und Erzählwelt wird hierdurch erleichtert (Bsp. 2). Allgemein zeigen die Fortführung, Suspendierung oder Wiederaufnahme manueller Tätigkeiten deutlich auf, wie die Teilnehmenden die Erzählung interpretieren und strukturieren. Die Fokussierung der TeilnehmerInnen auf das Erzählen erleichtert die gemeinschaftliche Ausarbeitung verschiedener Phasen und somit die Herstellung der Erzählung als soziales Ereignis, die über die Darstellung faktischer Inhalte hinausgeht (insbesondere Bsp. 2, vgl. Kallmeyer 1981).

Die Auszüge illustrieren, dass Alltagserzählungen in ihrer Struktur nicht einheitlich sind: Sie können klar von Beginn an als solche gerahmt werden und in Serien auftreten (Bsp. 2), oder aber als regulärer Redebeitrag beginnen und erst in der Folge zu einer Erzählung ausgearbeitet werden (Bsp. 1). Auch der Ausstieg aus dem Prozess des Erzählens ist selten an einem einzelnen Redebeitrag des Haupterzählers auszumachen, sondern wird gemeinsam hergestellt. Neben scherzhaften, fiktiven Weiterentwicklungen des Erzählten durch die Rezipienten (Bsp. 1) kann auch die gemeinsame narrative Nachverbrennung einer amüsanten Erzählung (Bsp. 2) auf deren Beendigung hinführen. Neben der Wiederaufnahme weiterer Aktivitäten (Bier einschenken, essen, Bsp. 1) kann auch die langsame Herunterstufung in hör- und sichtbarer Ausgestaltung (Bsp. 2) dazu genutzt werden, den während und für die Erzählung konstituierten Teilnehmerrahmen zu dekonstruieren.

Die Beteiligungsrollen, so zeigen diese Überlegungen, können nur noch ungenau mit den Bezeichnungen ErzählerIn und ZuhörerIn erfasst werden (vgl. etwa Quasthoff 2001, S. 1300): Vielmehr sind die HörerInnen gleichzeitig auch ZuschauerInnen und Mitakteure einer audio-visuellen (und möglicherweise auch über andere Sinneseindrücke wahrnehmbaren) Inszenierung, deren einzelne Elemente sie aufnehmen können, um dem vorherigen Sprecher zu antworten, das Erzählte im Rahmen einer affiliativen Handlung auszubauen oder um an Vorheriges anzuknüpfen, um schließlich selber das Wort zu ergreifen. Eine interaktionale Narratologie sollte es sich daher zur Aufgabe machen, Erzählpraktiken in möglichst vielfältigen, nicht-elizitierten und multimodalen Kontexten zu untersuchen, um die Bandbreite alltäglichen Erzählens zu systematisieren. Nur in einem solchen holistischen Zugang lässt sich die Komplexität der Koordination verschiedener Modalitäten, die alltägliches Erzählen in Ko-Präsenz charakterisiert, adäquat erfassen. 


\section{Multimodale Transkriptionskonventionen}

Sichtbare Handlungen sind nach den Konventionen von L. Mondada annotiert worden (für eine volle Version mit Beispielen s. https://franzoesistik.philhist.unibas.ch/ fileadmin/user_upload/franzoesistik/mondada_multimodal_conventions.pdf, s. auch Mondada 2018). $* *,++, \% \%, \$ \$$

Beschreibungen von sichtbaren Handlungen werden von zwei identischen Symbolen eingegrenzt, die mit dem Verbaltranskript bzw. den Pausen synchronisiert sind (jedem Teilnehmer ist ein Zeichen zugeordnet).

*--> $\quad$ Die Handlung wird in der/den folgenden Zeile(n) weitergeführt.

$\rightarrow 010$ Die Handlung wird bis zur Zeile 10 des Transkripts fortgeführt.

--->* Die Handlung wird bis zum Zeichen weitergeführt.

$>---\quad$ Die Handlung hat in einer vorherigen Zeile begonnen.

$>$ Die Handlung hat vor dem Auszug begonnen.

$-->>\quad$ Die Handlung wird bis über das Ende des Auszugs hinaus ausgeführt. Vorbereitung / Beginn der Handlung bzw. Bewegung.

Volle Ausstreckung oder Höhepunkt der Bewegung bzw. Handlung wird weiterhin ausgeführt.

,,, $\quad$ Retraktion der Handlung bzw. Bewegung.

jan Teilnehmer, der die jeweilige sichtbare Handlung ausführt.

\# Symbol, das die genaue Position eines Standbildes im Transkript anzeigt, \#2 mit jeweiliger Bildnummer.

\section{Literatur}

Bamberg, Michael: »Stories: Big or small. Why do we care?« In: Michael Bamberg (Hg.): Narrative State of the Art, Amsterdam 2007, S. 165-174.

Bergmann, Jörg: Klatsch. Zur Sozialform der diskreten Indiskretion. Berlin 1987.

Bergmann, Jörg/Luckmann, Thomas: »Reconstructive genres of everyday communication«. In: Uta M. Quasthoff (Hg.): Aspects of Oral Communication, Berlin/New York 1995, S. 289-304.

Bohle, Ulrike: Das Wort ergreifen, das Wort übergeben. Explorative Studie zur Rolle redebegleitender Gesten in der Organisation des Sprecherwechsels. Berlin 2007.

Duranti, Alessandro: »The audience as co-author. An introduction«. In: Text 6 (1986), S. 239-247.

Ehmer, Oliver: Imagination und Animation. Die Herstellung mentaler Räume durch animierte Rede. Berlin/New York 2011.

Fina, Anna de: »Group identity, narrative and self-representations«. In: Anna de Fina/Deborah Schiffrin/ Michael Bamberg (Hg.): Discourse and Identity. Cambridge 2006, S. 351-375.

Ford, Cecilia E./Fox, Barbara: »Multiple practices for constructing laughables«. In: Dagmar Barth-Weingarten/Elisabeth Reber/Margret Selting (Hg.): Prosody in Interaction. Amsterdam 2010, S. 338-368.

Georgakopoulou, Alexandra: »Small stories research«. In: Anna de Fina/Alexandra Georgakopoulou (Hg.): The Handbook of Narrative Analysis. Chichester 2015, S. 255-271.

Goodwin, Charles: Conversational Organization. Interaction between Speakers and Hearers. New York 1981.

Goodwin, Charles: »Notes on story structure and the organization of participation«. In: J. Maxwell Atkinson/John Heritage (Hg.): Structures of Social Action. Studies in Conversation Analysis. Cambridge 1984, S. 225-246.

Goodwin, Charles: »Audience diversity, participation and interpretation«. In: Text 6 (1986), S. 283-316.

Goodwin, Charles: »Narrative as Talk-in-Interaction«. In: Anna de Fina/Alexandra Georgakopoulou (Hg.): The Handbook of Narrative Analysis. Chichester 2015, S. 197-218.

Goodwin, Marjorie Harness: »Byplay: participant structure and framing of collaborative collusion«. In: Bernard Conein/Michel de Fornel/Louis Quére (Hg.): Les formes de la conversation. Analyse 
de l'action et analyse de la conversation. Colloque - Septembre 1987. Issy-les-Molineaux 1991, S. $155-180$.

Goodwin, Charles/Goodwin, Marjorie Harness: »Participation«. In: A. Duranti (Hg.): A Companion to Linguistic Anthropology. Oxford 2004, S. 222-243.

Gülich, Elisabeth: »Konventionelle Muster und kommunikative Funktionen von Alltagserzählungen«. In: Konrad Ehlich (Hg.): Erzählen im Alltag. Frankfurt a.M. 1980, S. 335-384.

Gülich, Elisabeth: »Alltägliches erzählen und alltägliches Erzählen«. In: Zeitschrift für germanistische Linguistik 36 (2008), S. 403-426.

Gülich, Elisabeth/Mondada, Lorenza: Konversationsanalyse: Eine Einführung am Beispiel des Französischen. Tübingen 2008.

Gumperz, John: »Contextualization revisited«. In: Peter Auer/Aldo DiLuzio (Hg.): The contextualization of language. Amsterdam 1992, S. 39-53.

Gunkel, Hermann: Die israelitische Literatur. Stuttgart 1925/1963.

Günthner, Susanne: »Beschwerdeerzählungen als narrative Hyperbeln«. In: Jörg Bergmann/Thomas Luckmann (Hg.): Kommunikative Konstruktion von Moral. Band 1: Struktur und Dynamik der Formen moralischer Kommunikation. Opladen/Wiesbaden 1999, S. 174-205.

Haddington, Pentti/Keisanen, Tiina/Mondada, Lorenza/Nevile, Maurice (Hg.): Multiactivity in Social Interaction. Beyond Multitasking. Amsterdam 2014.

Halkowski, Tim: »Realizing the illness: patients' narratives of symptom discovery«. In: John Heritage/ Douglas W. Maynard (Hg.): Communication in Medical Care. Interaction Between Primary Care Physicians and Patients. Cambridge 2007, S. 86-114.

Hausendorf, Heiko: »Interaktion im Raum Interaktionstheoretische Bemerkungen zu einem vernachlässigten Aspekt von Anwesenheit«. In: Arnulf Deppermann/Angelika Linke (Hg.): Sprache intermedial. Stimme und Schrift, Bild und Ton. Berlin 2010, S. 163-198.

Heinemann, Margot: »Textsorten des Alltags«. In: Klaus Brinker/Gerd Antos/Wolfgang Heinemann/Sven F. Sager (Hg.): Text- und Gesprächslinguistik. Ein internationales Handbuch zeitgenössischer Forschung. 1. Halbband. New York/Berlin 2000, S. 604-614.

Jefferson, Gail: »Sequential aspects of storytelling in conversation«. In: Jim Schenkein (Hg.): Studies in the Organization of Conversation. New York 1978, S. 79-112.

Jefferson, Gail: »On the sequential organization of troubles-talk in ordinary conversation«. In: Social Problems 35 (1988), S. 418-441.

Kallmeyer, Werner: »>(expressif) eh ben dis donc, hein pas bien< Zur Beschreibung von Exaltation als Interaktionsmodalität«. In: Rolf Kloepfer (Hg.): Bildung und Ausbildung in der Romania. Band I: Literaturgeschichte und Texttheorie. München 1979, S. 549-568.

Kallmeyer, Werner: »Gestaltungsorientiertheit in Alltagserzählungen«. In: Rolf Kloepfer/Gisela JanetzkeDillner (Hg.): Erzählung und Erzählforschung im 20. Jahrhundert. Stuttgart 1981, S. 409-429.

Keevallik, Leelo: »The interdependence of bodily demonstrations and clausal syntax«. In: Research on Language \& Social Interaction 46 (2013), S. 1-21.

Keppler, Angela: Tischgespräche. Über Formen kommunikativer Vergemeinschaftung am Beispiel der Konversation in Familien. Frankfurt am Main 1994.

Kotthoff, Helga: Spaß verstehen. Zur Pragmatik von konversationellem Humor. Tübingen 1998.

Lerner, Gene H.: »Assisted storytelling: Deploying shared knowledge as a practical matter«. In: Qualitative Sociology 15 (1992), S. 247-271.

Lerner, Gene H.: »On the >semi-permeable< character of grammatical units in conversation. Conditional entry into the turn space of another speaker«. In: Elinor Ochs/Emanuel A. Schegloff/Sandra A. Thompson (Hg.): Interaction and grammar. Cambridge/New York 1996, S. 238-276.

Lerner, Gene H.: »Turn-sharing. The coral co-production of talk-in-interaction«. In: Cecilia E. Ford/ Barbara A. Fox/Sandra A. Thompson (Hg.): The Language of Turn and Sequence. New York 2002, S. $225-256$.

Lerner, Gene H.: »Collaborative turn sequences«. In: Gene H. Lerner (Hg.): Conversation Analysis. Studies from the First Generation. Amsterdam 2004, S. 225-256.

Lucius-Hoene, Gabriele/Deppermann, Arnulf: »Narrative Identität und Positionierung «. In: Gesprächsforschung. Online-Zeitschrift zur verbalen Interaktion 5 (2004), S. 166-183.

Mandelbaum, Jennifer: »Couples sharing stories«. In: Communication Quarterly 35 (1987), S. 144-170.

Mandelbaum, Jenny: »Storytelling in conversation«. In: Jack Sidnell/Tanya Stivers (Hg.): The Handbook of Conversation Analysis. Chichester 2013, S. 492-507.

Mondada, Lorenza: »Multimodal interaction«. In: Cornelia Müller/Alan J. Cienki/Ellen Fricke/Silva H. Ladewig/David McNeill/Sedinha Tessendorf (Hg.): Body - Language - Communication. An International Handbook on Multimodality in Human Interaction, Volume 1. Berlin/Boston 2013, S. 577-589. 
Mondada, Lorenza: »The local constitution of multimodal resources for social interaction«. In: Journal of Pragmatics 65 (2014a), S. 137-156.

Mondada, Lorenza: »The temporal orders of multiactivity. Operating and demonstrating in the surgical theatre «. In: Pentti Haddington/Tiina Keisanen/Lorenza Mondada/Maurice Nevile (Hg.): Multiactivity in Social Interaction. Beyond Multitasking. Amsterdam 2014b, S. 33-76.

Mondada, Lorenza: »Multiple temporalities of language and body in interaction. Challenges for transcribing multimodality«. In: Research on Language and Social Interaction 51 (2018), S. 85-106.

Müller, Cornelia: »On the gestural creation of narrative structure: A case study of a story told in a conversation«. In: Monica Rector/Isabella Poggi/Nadine Trigo (Hg.): Gestures. Meaning and use. Porto 2003, S. 259-265.

Nevile, Maurice: »Talking without overlap in the airline cockpit. Precision timing at work«. In: Text \& Talk 27 (2007), S. 225-249.

Norrick, Neal R.: »Twice-told tales: Collaborative narration of familiar stories«. In: Language in Society 26 (1997), S. 199-220.

Ochs, Elinor/Capps, Lisa: Living Narrative. Creating lives in Everyday Storytelling. Cambridge 2001.

Ochs, Elinor/Taylor, Carolyn: »The >father knows best< dynamic in family dinner narratives« In: Kira Hall/ Mary Bucholtz (Hg.): Gender Articulated. Language and the Socially Constructed Self. New York 1995, S. 97-121.

Oloff, Florence: »Embodied withdrawal after overlap resolution«. In: Journal of Pragmatics 46 (2013), S. $139-156$

Olsher, David: »Talks and gesture. The embodied completion of sequential actions in spoken interaction«. In: Rod Gardner/Johannes Wagner (Hg.): Second Language Conversations. London/New York 2004, S. 221-245.

Quasthoff, Uta M.: »Erzählen als interaktive Gesprächsstruktur«. In: Klaus Brinker/Gerd Antos/Wolfgang Heinemann/Sven F. Sager (Hg.): Text- und Gesprächslinguistik. Ein internationales Handbuch zeitgenössischer Forschung. 2. Halbband. Berlin/New York 2001, S. 1293-1309.

Ruusuvuori, Johanna/Peräkylä, Anssi: »Facial and verbal expressions in assessing stories and topics«. In: Research on Language and Social Interaction 42 (2009), S. 377-394.

Sacks, Harvey: »Das Erzählen von Geschichten innerhalb von Unterhaltungen«. In: Rolf Kjolseth/Fritz Sack (Hg.): Zur Soziologie der Sprache. Opladen 1971, S. 307-314.

Sacks, Harvey: »Some considerations of a story told in ordinary conversations«. In: Poetics 15 (1986), S. 127-138.

Sacks, Harvey: Lectures on Conversation. Volume II. Oxford, UK 1995.

Sacks, Harvey/Schegloff, Emanuel/Jefferson, Gail: »A simplest systematics for the organization of turntaking for conversations«. In: Language 50 (1974), S. 696-735.

Schegloff, Emanuel: »Recycled turn beginnings. A precise repair mechanism in conversation's turn-taking organization«. In: Graham Button/John R. E. Lee (Hg.): Talk and Social Organization. Clevedon 1987, S. 70-85.

Schegloff, Emanuel: »Body torque«. In: Social Research 65 (1998), S. 535-596.

Schmitt, Reinhold: »Positionspapier: Multimodale Interaktionsanalyse«. In: Ulrich Dausendschön-Gay/ Elisabeth Gülich/Ulrich Krafft (Hg.): Ko-Konstruktionen in der Interaktion. Die gemeinsame Arbeit an Äußerungen und anderen sozialen Ereignissen. Bielefeld 2015, S. 43-51.

Schumann, Elke/Gülich, Elisabeth/Lucius-Hoene, Gabriele/Pfänder, Stefan (Hg.): Wiedererzählen. Formen und Funktionen einer kulturellen Praxis. Bielefeld 2015.

Schütte, Wilfried: »Alltagsgespräche«. In: Klaus Brinker/Gerd Antos/Wolfgang Heinemann/Sven F. Sager (Hg.): Text- und Gesprächslinguistik. Ein internationales Handbuch zeitgenössischer Forschung. 2. Halbband. Berlin/New York 2001, S. 1485-1492.

Schwitalla, Johannes: »Gespräche über Gespräche. Nach- und Nebengespräche über ausgeblendete Aspekte einer Interaktion«. In: Gesprächsforschung. Online-Zeitschrift zur verbalen Interaktion 7 (2006), S. 229-247.

Selting, Margret: »Complaint stories and subsequent complaint stories with affect displays«. In: Journal of Pragmatics 44 (2012), S. 387-415.

Selting, Margret: »The display and management of affectivity in climaxes of amusing stories«. In: Journal of Pragmatics 111 (2017), S. 1-32.

Selting, Margret/Auer, Peter/Barth-Weingarten, Dagmar et al: »Gesprächsanalytisches Transkriptionssystem 2 (GAT 2)«. In: Gesprächsforschung - Online-Zeitschrift zur verbalen Interaktion 10 (2009), S. 353-402.

Sidnell, Jack: »Coordinating gesture, talk, and gaze in reenactments«. In: Research on Language and Social Interaction 39 (2006), S. 377-409. 
Sidnell, Jack: Conversation Analysis. An Introduction. Chichester/Malden 2010.

Siromaa, Maargit: »Resonance in conversational second stories: a dialogic resource for stance taking «. In: Text \& Talk 32 (2012), S. 525-545.

Stec, Kashmiri/Huiskes, Mike/Redeker, Gisela: »Multimodal quotation. Role shift practices in spoken narratives«. In: Journal of Pragmatics 104 (2016), S. 1-17.

Stivers, Tanya: »Stance, alignment, and affiliation during storytelling: When nodding is a token of affiliation«. In: Research on Language and Social Interaction 41 (2008), S. 31-57.

Stukenbrock, Anja: »Pointing to an >empty< space. Deixis am Phantasma in face-to-face interaction«. In: Journal of Pragmatics 74 (2014), S. 70-93.

Thompson, Sandra A./Suzuki, Ryoko: »Reenactments in conversation. Gaze and recipiency«. In: Discourse Studies 16 (2014), S. 816-846. 